Preprint typeset using LAT $_{\mathrm{E}} \mathrm{X}$ style emulateapj v. 08/22/09

\title{
ENERGETIC GAMMA RADIATION FROM RAPIDLY ROTATING BLACK HOLES
}

Kouichi Hirotani, AND Hung-Yi Pu

Academia Sinica, Institute of Astronomy and Astrophysics (ASIAA), PO Box 23-141, Taipei, Taiwan

\begin{abstract}
Supermassive black holes are believed to be the central power house of active galactic nuclei. Applying the pulsar outer-magnetospheric particle accelerator theory to black-hole magnetospheres, we demonstrate that an electric field is exerted along the magnetic field lines near the event horizon of a rotating black hole. In this particle accelerator (or a gap), electrons and positrons are created by photon-photon collisions and accelerated in the opposite directions by this electric field, efficiently emitting gamma-rays via curvature and inverse-Compton processes. It is shown that a gap arises around the null charge surface formed by the frame-dragging effect, provided that there is no current injection across the gap boundaries. The gap is dissipating a part of the hole's rotational energy, and the resultant gamma-ray luminosity increases with decreasing plasma accretion from the surroundings. Considering an extremely rotating supermassive black hole, we show that such a gap reproduces the significant very-high-energy (VHE) gamma-ray flux observed from the radio galaxy IC 310, provided that the accretion rate becomes much less than the Eddington rate particularly during its flare phase. It is found that the curvature process dominates the inverse-Compton process in the magnetosphere of IC 310, and that the observed power-law-like spectrum in VHE gamma-rays can be explained to some extent by a superposition of the curvature emissions with varying curvature radius. It is predicted that the VHE spectrum extends into higher energies with increasing VHE photon flux.

Subject headings: black hole physics — galaxies: individual (IC 310) — gamma rays: stars — magnetic fields - methods: analytical — methods: numerical
\end{abstract}

\section{INTRODUCTION}

The MAGIC (Major Atmospheric Gamma-ray Imaging Cherenkov) telescopes recently reported on gamma-ray observations of the radio galaxy IC 310 Aleksic et al. 2014b), which presumably harbors a black hole (BH) with 0.1-0.7 billion solar masses (Aleksic et al. 2014b; McElroy 1995; Simien \& Prugniel 2002). MAGIC detected a powerful flare in very high energies (VHE) between $70 \mathrm{GeV}$ and $8 \mathrm{TeV}$ and revealed that the flare flickers with a doubling time scale as short as 4.8 minutes. Ruling out other possibilities, the MAGIC team inferred that a substantial portion of the flaring photons originated from a compact region as small as one fifth of the typical $\mathrm{BH}$ radius, or the Schwarzschild radius, $2 r_{\mathrm{g}}=3 \times 10^{14} M_{9} \mathrm{~cm}$, where $M_{9}=M /\left(10^{9} M_{\odot}\right)$ denotes the $\mathrm{BH}$ mass in billion solar masses.

To interpret this amazing finding of a unique subhorizon phenomenon, we apply the pulsar vacuumgap model (Cheng et al. 1986a) to the BH magnetosphere of IC 310, extending the method developed by Beskin et al. (1992) and Hirotani \& Okamoto (1998, hereafter HO98). It is known that the rotational energy of a $\mathrm{BH}$ can be electromagnetically extracted with spin-down luminosity (Blandford \& Znajek 1977; Koide et al. 2002)

$$
L_{\mathrm{sd}}=\Omega_{\mathrm{F}}\left(\omega_{\mathrm{H}}-\Omega_{\mathrm{F}}\right) B_{\perp}^{2} r_{\mathrm{H}}^{4} / c,
$$

where $\Omega_{\mathrm{F}}$ denotes the angular frequency of the rotating magnetic field lines, $\omega_{\mathrm{H}}=a c /\left[2\left(G M c^{-2}\right) r_{\mathrm{H}}\right]$ the angular frequency of the rating $\mathrm{BH}, B_{\perp}$ the normal component of the magnetic field threading the event horizon, $r_{\mathrm{H}}$ the radius of the horizon, and $c$ the speed of light. A portion

Electronic address: hirotani@tiara.sinica.edu.tw of such a spin-down energy can be dissipated as photon emissions within the magnetosphere.

In $\S 2.1$, we outline previous BH gap models, arranging their electrodynamic properties (and hidden philosophies) according to the Poisson equation for the noncorotational potential. Then in $\S$ 3. we point out that the magnetosphere of IC 310 is usually filled with copious plasmas that quench a $\mathrm{BH}$ gap, and that a $\mathrm{BH}$ gap can be switched on if the mass accretion rate is halved from the time-averaged value. We formulate the $\mathrm{BH}$ gap in $\S$ 4, and apply the model to IC 310 in $\S$ 5. We highlight the difference among current $\mathrm{BH}$ gap models in the final section.

\section{GAP POSITION}

In this section, we investigate the plausible position of particle accelerators in a black hole magnetosphere.

\subsection{The null charge surface}

When magnetized plasmas are accreting onto an astrophysical BH, the self-gravity of the plasma particles and the electromagnetic field little affects the spacetime geometry. Thus, around a rotating $\mathrm{BH}$, the background geometry is described by the Kerr metric (Kerr 1963). In the Boyer-Lindquist coordinates, it becomes (Boyer \& Lindquist 1967)

$$
d s^{2}=g_{t t} d t^{2}+2 g_{t \varphi} d t d \varphi+g_{\varphi \varphi} d \varphi^{2}+g_{r r} d r^{2}+g_{\theta \theta} d \theta^{2}
$$

where

$$
\begin{gathered}
g_{t t} \equiv-\frac{\Delta-a^{2} \sin ^{2} \theta}{\Sigma} c^{2}, \quad g_{t \varphi} \equiv-\frac{2\left(G M / c^{2}\right) a r \sin ^{2} \theta}{\Sigma} c, \\
g_{\varphi \varphi} \equiv \frac{A \sin ^{2} \theta}{\Sigma}, \\
g_{r r} \equiv \frac{\Sigma}{\Delta}, \quad g_{\theta \theta} \equiv \Sigma ;
\end{gathered}
$$


$\Delta \equiv r^{2}-2\left(G M / c^{2}\right) r+a^{2}, \Sigma \equiv r^{2}+a^{2} \cos ^{2} \theta, A \equiv$ $\left(r^{2}+a^{2}\right)^{2}-\Delta a^{2} \sin ^{2} \theta$, and $a \equiv J /(M c), c$ denotes the speed of light, $M$ the mass of the $\mathrm{BH}, J$ the $\mathrm{BH}$ 's angular momentum. At the event horizon, $\Delta$ vanishes, giving $r_{\mathrm{H}} \equiv r_{\mathrm{g}}+\sqrt{r_{\mathrm{g}}{ }^{2}-a^{2}}$ as the horizon radius, where $r_{\mathrm{g}} \equiv$ $G M c^{-2}$.

In the same manner as in pulsar magnetospheres, the Gauss's law, $\nabla_{\mu} F^{t \mu}=(4 \pi / c) \rho$, gives the Poisson equation for the non-corotational potential $\Psi$ (Hirotani 2006),

$$
-\frac{c^{2}}{\sqrt{-g}} \partial_{\mu}\left(\frac{\sqrt{-g}}{\rho_{\mathrm{w}}^{2}} g^{\mu \nu} g_{\varphi \varphi} \partial_{\nu} \Psi\right)=4 \pi\left(\rho-\rho_{\mathrm{GJ}}\right)
$$

where the Greek indices run over $t, r, \theta, \varphi, \sqrt{-g}=$ $\sqrt{g_{r r} g_{\theta \theta} \rho_{\mathrm{w}}^{2}}=c \Sigma \sin \theta, \rho_{\mathrm{w}}^{2} \equiv g_{t \varphi}^{2}-g_{t t} g_{\varphi \varphi}$, and $\rho$ the real charge density; the general relativistic GJ charge density is defined as (Goldreich \& Julian 1969; Mestel 1971; Hirotani 2006)

$$
\rho_{\mathrm{GJ}} \equiv \frac{c^{2}}{4 \pi \sqrt{-g}} \partial_{\mu}\left[\frac{\sqrt{-g}}{\rho_{\mathrm{w}}^{2}} g^{\mu \nu} g_{\varphi \varphi}\left(\Omega_{\mathrm{F}}-\omega\right) F_{\varphi \nu}\right] .
$$

The magnetic-field-aligned electric field can be computed by $E_{\|} \equiv(\boldsymbol{B} / B) \cdot \boldsymbol{E}=(\boldsymbol{B} / B) \cdot(-\nabla \Psi)$.

If $\rho$ deviates from $\rho_{\mathrm{GJ}}$ in any region, $E_{\|}$is exerted there along $\boldsymbol{B}$. Once $E_{\|}$arises, positive and negative charges migrate in opposite directions. For the magnetosphere to be force-free outside the gap, $\rho$ should match $\rho_{\mathrm{GJ}}$ at the boundaries. Since $\rho$ has opposite signs at the two boundaries, $E_{\|}$should arise around the null charge surface, where $\rho_{\text {GJ }}$ vanishes, in the same way as pulsars (Cheng et al. 2000; Romani 1996). On these grounds, we consider that the null surface is a natural place for a particle accelerator to arise.

In figure 1, we plot the distribution of the null surface as the thick red solid curve. In the left panel, we assume that the magnetic field is split-monopole, adopting $A_{\varphi} \propto-\cos \theta$ as the magnetic flux function (Michel 1973). In the right panel, on the other hand, we assume a parabolic magnetic field line with $A_{\varphi} \propto$ $r(1-\cos \theta)$ on the poloidal plane (i.e., $r-\theta$ plane). We adopt $\Omega_{\mathrm{F}}=0.3 \omega_{\mathrm{H}}$ for both cases (McKinney et al. 2012; Beskin \& Zheltoukhov 2013). It follows that the null surface distributes nearly spherically, irrespective of the poloidal magnetic field configuration. This is because its position is essentially determined by the condition $\omega=\Omega_{\mathrm{F}}\left(A_{\varphi}\right)$, because $\omega$ has weak dependence on $\theta$ near the horizon, and because we assume $\Omega_{\mathrm{F}}$ is constant for $A_{\varphi}$ for simplicity. If $\Omega_{\mathrm{F}}$ decreases (or increases) toward the polar region, the null surface shape becomes prolate (or oblate).

The field angular frequency $\Omega_{\mathrm{F}}$ is, indeed, deeply related to the accretion conditions. For instance, in order to get adequate jet efficiency from the magnetic field in the funnel above the black hole for a radio loud active galactic nuclei (AGN), one needs significant flux compression from the lateral boundary imposed by the accretion flow and corona (McKinney et al. 2012; Sikora \& Begelman 2013). These lateral boundary conditions and the plasma injection process will modify $\Omega_{\mathrm{F}}$ in the funnel to $-0.10 \omega_{\mathrm{H}}<\Omega_{\mathrm{F}}<0.35 \omega_{\mathrm{H}}$ (McKinnev et al. 2012; Beskin \& Zheltoukhov 2013). For illustrative purposes, we simplify this behavior by choosing a constant $\Omega_{\mathrm{F}}=0.3 \omega_{\mathrm{H}}$ for $A_{\varphi}$. We acknowl-

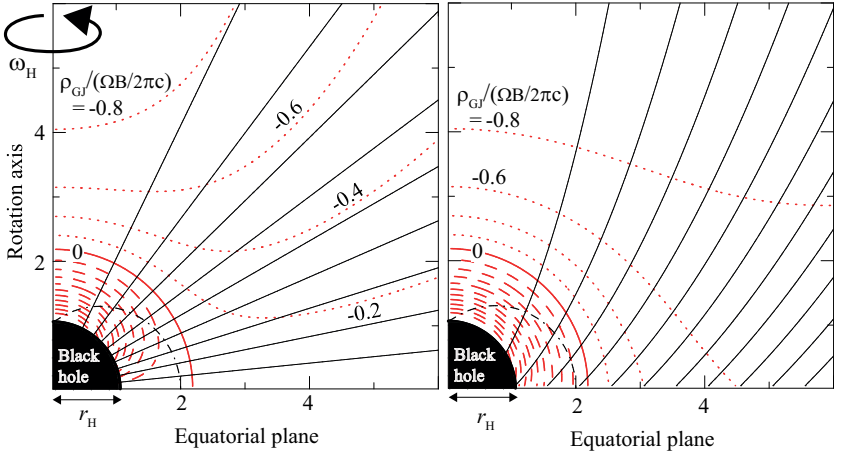

FIG. 1.- Distribution of the null surface (red thick solid curve) on the poloidal plane in the Boyer-Lindquist coordinates. The axes are in $r_{\mathrm{g}}=G M c^{-2}$ unit. The black hole (filled black region in the lower left corner) rotates rapidly with spin parameter $\mathrm{a}=0.998 r_{\mathrm{g}}$ around the ordinate. The contours of the dimensionless GoldreichJulian charge density are plotted with the red dashed curves (for positive values) and the red dotted ones (for negative values as labeled). The black dash-dotted curve denotes the static limit, within which the rotational energy of the hole is stored. The black solid curves denote the magnetic field lines. The left panel is the result for the case of a split-monopole magnetic field, and the right one for a parabolic field.

edge that this is not a self consistent solution, but we believe that it is sufficient for demonstrating the physical process that we propose. In $\S[5.9$, we consider the cases of different $\Omega_{\mathrm{F}}$ and compare the results.

\subsection{The stagnation surface}

In addition to the null surface discussed above, a stagnation surface is also considered to be a plausible place for particle accelerators to arise. Since both inflows and outflows start from the stagnation surface in magnetohydrodynamics (MHD), we can expect that the plasma density becomes low, thereby leading to a nonvanishing $E_{\|}$there. Levinson \& Rieger (2011, LR11) and Broderick \& Tchekhovskoy (2011, BT15) examined this possibility. In this subsection, we examine only the position of the stagnation surface, leaving its electrodynamical plausibility as a particle accelerator site to $\S 2.3$.

In a stationary and axisymmetric black hole magnetosphere, MHD inflows and outflows start from the twodimensional surface on which

$$
k_{0}^{\prime}=0
$$

holds (Takahasi et al. 1990), where

$$
k_{0} \equiv-g_{t t}-2 g_{t \varphi} \Omega_{\mathrm{F}}-g_{\varphi \varphi} \Omega_{\mathrm{F}}{ }^{2},
$$

and the prime denotes the derivative along the poloidal magnetic field line. The condition (7) is equivalent with imposing a balance among the gravitational, centrifugal, and Lorentz forces on the poloidal plane. In figure 2, we plot the contours of $k_{0}$ for split-monopole and parabolic poloidal magnetic field lines. Far away from the horizon, we obtain $k_{0} \approx c^{2}-\varpi^{2} \Omega_{\mathrm{F}}{ }^{2}$, where $\varpi$ denotes the distance from the rotation axis. Thus, at large $\varpi$, the potential $k_{0}$ becomes negative, as denoted by the red dotted contours on the right part of each panel. Note that $k_{0}$ vanishes at $\varpi \approx c / \Omega_{\mathrm{F}}$. This two-dimensional surface is called the outer light surface and depicted by the thick red solid curve in figure 2, If the general-relativistic effects (i.e., redshift and frame-dragging effects) were negligible, and if $\Omega_{\mathrm{F}}$ is constant for the magnetic flux function $A_{\varphi}$, the 
outer light surface would distribute cylindrically, as in pulsar magnetospheres. Outside the outer light surface, plasma particles must flow outwards, if they are frozenin to the magnetic field. Inside the outer light surface, $k_{0}$ becomes positive, as denoted by the dashed contours. Further inside, close to the horizon, $k_{0}$ begins to decrease again due to the strong gravity of the hole. As a result, another light surface, where $k_{0}$ vanishes, appears, as depicted by the thick red solid curve near the horizon. Inside this inner light surface, plasma particles must flow inwards if they are frozen-in to the magnetic field.

As demonstrated by Takahasi et al. (1990), a stationary and axisymmetric MHD flow flows from a greater $k_{0}$ region to a smaller $k_{0}$ one. It follows that both the inflows and outflows start from the two-dimensional surface on which $k_{0}$ maximizes along the poloidal magnetic field line. Thus, putting $k_{0}{ }^{\prime}=0$, we obtain the position of the stagnation surface. If figure 2, we plot the stagnation surface as the thick green solid curve. It is clear that the stagnation surface is located at $r<5 r_{\mathrm{g}}$ in the lower latitudes but at $r>5 r_{\mathrm{g}}$ in the higher latitudes, irrespective of the magnetic field line configuration, as long as $\Omega_{\mathrm{F}}$ is a good fraction of $\omega_{\mathrm{H}}$, e.g., $0.25 \omega_{\mathrm{H}}<\Omega<0.50 \omega_{\mathrm{H}}$. It is, however, noteworthy that the numerical simulations (McKinnev et al. 2012) and a supporting analytical work (Beskin \& Zheltoukhov 2013) claimed $-0.10 \omega_{\mathrm{H}}<\Omega<0.35 \omega_{\mathrm{H}}$ in the funnel to represent radio-loud AGN. As the poloidal field geometry changes from radial to parabolic, the stagnation surface moderately moves away from the rotation axis in the higher latitudes.

This analytical result was confirmed by general relativistic (GR) MHD simulations (McKinney et al. 2006; Broderick \& Tchekhovskov 2011). In these numerical works, the stagnation surface is time-dependent but stably located at $5-10 r_{\mathrm{g}}$ with a prolate shape, as depicted in figure 2, In addition, the position of the stagnation surface changes as a function of the accretion rate and other numerical settings such as the initial and boundary conditions. However, it should be emphasized that these changes are caused merely through the poloidal magnetic field configuration and $\Omega_{\mathrm{F}}=\Omega_{\mathrm{F}}\left(A_{\varphi}\right)$, the latter of which defines the potential $k_{0}$.

To examine the Poisson equation (5), it is worth noting that the distribution of $\rho_{\mathrm{GJ}}$ has relatively small dependence on the magnetic field geometry near the horizon, because its distribution is essentially governed by the space-time dragging effect, as indicated by the red solid, dashed, and dotted contours in figure 1 for splitmonopole and parabolic fields. As a result, the gap solution little depends on the magnetic field line configuration, particularly in the higher latitudes, forming a striking contrast to pulsar outer-gap models, in which the field line configuration defines $\rho_{\mathrm{GJ}}$.

As for $k_{0}=k_{0}(r, \theta)$, it has no dependence on the field geometry, as long as $\Omega_{\mathrm{F}}$ is constant for all the field lines. In this particular case, the stagnation surface distributes more or less similarly for split-monopole and parabolic fields, as figure 2 indicates. Thus, in what follows, we assume a split-monopole field and adopt a constant $\Omega_{\mathrm{F}}=\Omega_{\mathrm{F}}\left(A_{\varphi}\right)$ for simplicity, as described in the left panels of figures 1 and 2. However, it is noteworthy that the lateral boundary conditions and the plasma injection in the tenuous funnel alter both the magnetic-

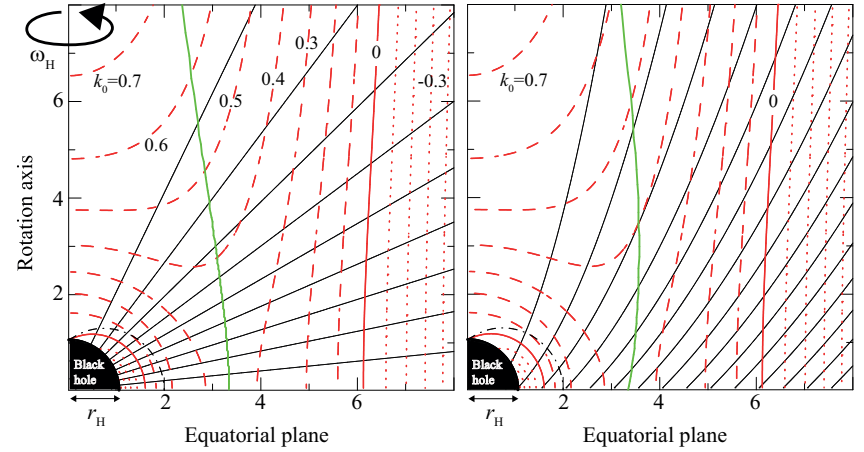

FIG. 2.- Distribution of the stagnation surface (thick green solid curve) on the poloidal plane. Similar figure as figure 1 but equi- $k_{0}$ potential curves are depicted with the dashed curves (for positive values) and dotted ones (for negative values). The thick, red solid curves denote the outer and inner light surfaces where $k_{0}$ vanishes. Stationary MHD flows start from the maximum- $k_{0}$ point along the individual magnetic field lines (Takahasi et al. 1990). The left panel represents the case of a split-monopole magnetic field, while the right one a parabolic field.

field distribution near the event horizon and $\Omega_{\mathrm{F}}$ in an actual solution (Phinney 1983; McKinney et al. 2012; Beskin \& Zheltoukhov 2013).

\subsection{Electrodynamical consideration of gap position}

Let us leave the geometrical argument of the stagnation surface and turn to the electrodynamics of a gap, which may be formed around the null surface or the stagnation surface. For this sake, we must consider the Poisson equation (5) for the non-corotational potential $\Psi$.

If the full gap width $w$ along the magnetic field line is short compared to the meridional and azimuthal dimensions of the gap, only the derivatives along the poloidal magnetic field line remains in equation (5). Noting that the gravitational forces are negligible compared to the electrodynamical forces in the gap (except for the intimate vicinity of the horizon), we can reduce equation (5) into

$$
\frac{d E_{\|}}{d s}=4 \pi\left(\rho-\rho_{\mathrm{GJ}}\right),
$$

where $s$ denotes the distance along the poloidal magnetic field line measured outward in the Boyer-Lindquist coordinates. We define $s=0$ to indicate the null surface, $r=r_{0}$. Thus, the position $s$ that satisfies $r_{0}+s=r_{\mathrm{H}}$ represents the event horizon, because we adopt radial magnetic field lines on the poloidal plane. Both the inner boundary (at $s=s_{1}$ ) and the outer boundary (at $s=s_{2}$ ) are free boundaries and are determined as a function of the accretion rate by the procedure to be described in $\S$ 4.5. If the real charge density $\rho$ is greater (or smaller) than $\rho_{\mathrm{GJ}}, E_{\|}$increases (or decreases) outwards.

The created $e^{ \pm}$pairs are separated by $E_{\|}$in the gap. Without loss of any generality, we can assume $E_{\|}<0$ in the gap, which is possible if $\boldsymbol{B} \cdot \boldsymbol{L}>0$ (or exactly speaking, if $A_{\varphi, \theta}>0$ ) in the northern hemisphere near the horizon, where $\boldsymbol{L}$ denotes the BH's angular momentum vector. Note that the sign of $E_{\|}$changes from pulsar outer gaps, because the null surface is formed by the frame dragging instead of the convex topology of the poloidal magnetic field lines. The negative $E_{\|}$results in an outwardly decreasing dimensionless charge density, $\rho /(\Omega B / 2 \pi c)$. Since the total current per magnetic flux tube conserves, the sum of positronic and electronic 
charge densities per magnetic flux tube also conserves. Thus, pair production per unit length, $d(\rho / B) / d s$, becomes more or less constant for $s$, provided that the background soft photon density is roughly homogeneous, as demonstrated in figure 5 of Hirotani \& Shibata (1999b). We therefore assume that $\rho / B$ decreases linearly with $s$ in the present paper. In general, to analyze the spatial dependence of $\rho / B$, we must solve the Boltzmann equations of electrons, positrons, and photons simultaneously together with the Poisson equation (9), as Hirotani \& Shibata (1999b) did for pulsar magnetospheres.

In the present paper, we employ a simplified model such that $\rho / B$ decreases with increasing $s$ linearly as

$$
\frac{\rho(s)}{B(s)}=j \frac{\rho_{\mathrm{GJ}}\left(s_{2}\right)}{B\left(s_{2}\right)} \frac{2 s-s_{1}-s_{2}}{s_{2}-s_{1}},
$$

where $0<j \leq 1$. Note that $s=s_{2}$ denotes the outer boundary and that $\rho\left(s_{2}\right)$ is negative. If we put $j=0$, we obtain a vacuum gap. In a non-vacuum gap, $j \neq 0, E_{\|}$is partially screened by the created and polarized pairs. If $j=1$, we obtain $\rho\left(s_{2}\right)=\rho_{\mathrm{GJ}}\left(s_{2}\right)$. A super-GJ current, $j>1$, is prohibited, as will be discussed in $\S 6.3 .2$

\subsubsection{Vacuum gap around the null surface}

Let us begin with the vacuum case, $j=0$, which leads to $\rho=0$. The Poisson equation (9) shows $d E_{\|} / d s>$ 0 (or $d E_{\|} / d s<0$ ) holds in the outer (or inner) part of the gap, because $\rho-\rho_{\mathrm{GJ}}=-\rho_{\mathrm{GJ}}$ becomes positive (or negative) in the outer (or inner) part of the gap. Thus, we obtain $E_{\|}<0$ in the gap, and find that $\left|E_{\|}\right|$ maximizes at the point where $\rho$ matches $\rho_{\mathrm{GJ}}$, which is realized at the null surface, $s=0$, in a vacuum gap. Outside the gap, on the other hand, to meet the forcefree condition, $\rho$ should match $\rho_{\mathrm{GJ}}$. It results in a jump of $d E_{\|} / d s$ at the boundaries, which should be compensated by the surface charge there.

For analytical purpose, in this particular subsection ( $\S 2.3 .1)$, we assume that the full gap width, $w$, is much small compared to $r_{\mathrm{g}}$, and expand equation (9) around the null surface $(s=0)$ to obtain

$$
\frac{d E_{\|}}{d s}=-4 \pi \rho_{\mathrm{GJ}}{ }^{\prime} s,
$$

where $\rho_{\mathrm{GJ}}{ }^{\prime}$ denotes the derivative of $\rho_{\mathrm{GJ}}$ at $s=0$. Imposing $E_{\|}=0$ at both boundaries $(s= \pm w / 2)$, we obtain

$$
E_{\|}(s)=2 \pi \rho_{\mathrm{GJ}}{ }^{\prime}\left[(w / 2)^{2}-s^{2}\right] .
$$

The distribution of $\rho / B, \rho_{\mathrm{GJ}} / B$, and $E_{\|}$are illustrated in figure 3. As the gap width $w$ increases, $\left|E_{\|}\right|$increases quadratically. Integrating equation (12) over $s$ from $s_{1}=$ $-w / 2$ to $s_{2}=+w / 2$, we obtain the electric potential drop in the gap,

$$
V_{\text {gap }}=\frac{8}{3} \pi \rho_{\mathrm{GJ}}{ }^{\prime}(w / 2)^{3} \approx\left(\frac{w / 2}{r_{\mathrm{H}}}\right)^{3}(\mathrm{EMF}),
$$

where the electromotive force exerted on the hemisphere of the horizon is given by

$$
\mathrm{EMF} \equiv \frac{\Omega_{\mathrm{F}}}{c} r_{\mathrm{H}}^{2} B
$$

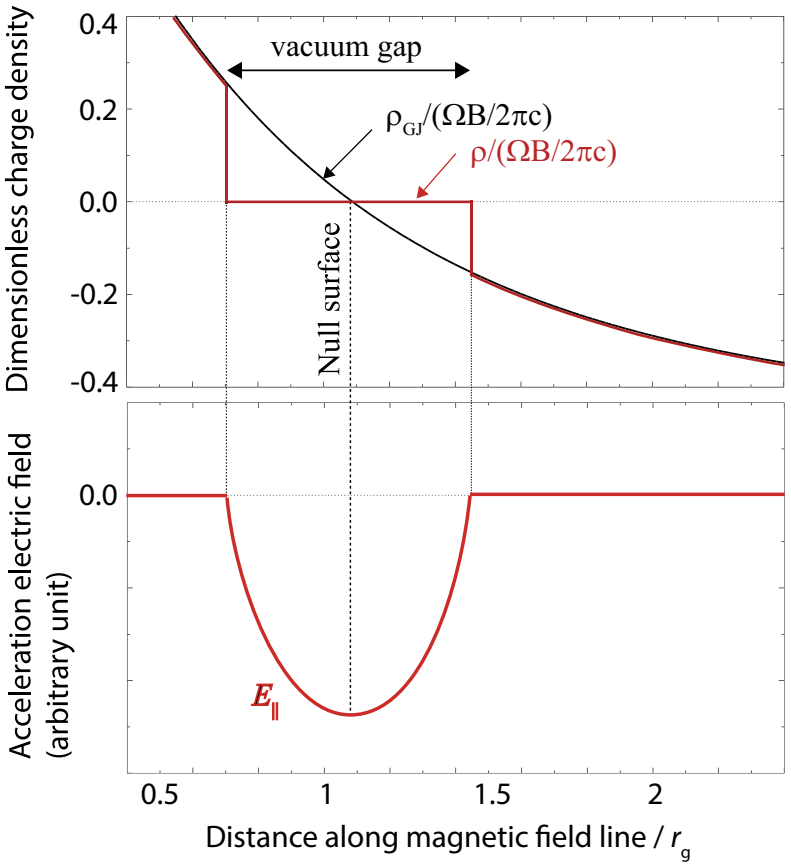

FIG. 3.- Distribution of the charge density and the magneticfield-aligned electric field along the magnetic field. A vacuum gap is assumed. In the upper panel, the black solid curve represents the Goldreich-Julian charge density per magnetic flux along the splitmonopole magnetic field line at $\theta=15^{\circ}$, while the red solid curve does the dimensionless real charge density per magnetic flux. In the lower panel, the resultant $E_{\|}$is illustrated so that the distribution of the $\rho-\rho_{\mathrm{GJ}}$ sign may be reflected. Since the gap closure condition $(\S 4.5)$ cannot be satisfied for $\rho=0$, we cannot determine the full gap width $w$. Thus, we can only illustrate the $E_{\|}$distribution by hand for a vacuum gap.

and

$$
\rho_{\mathrm{GJ}}{ }^{\prime} \approx \frac{\Omega_{\mathrm{F}} B}{2 \pi c} \frac{1}{r_{\mathrm{H}}}
$$

is used. Although we expand $\rho_{\text {GJ }}$ around $s=0$, the argument on $V_{\text {gap }}$ is basically valid even when $w>r_{\mathrm{g}}$, because $\rho_{\mathrm{GJ}}$ changes from 0 (at the null surface) to $\approx$ $\left(\omega_{\mathrm{H}}-\Omega_{\mathrm{F}}\right) B /(2 \pi c)$ (at the horizon) in any case.

If we assume some $w$, we can solve $E_{\|}(s)$ with equation (9). For a vacuum gap, there is no photon flux that is needed to realize the pair creation cascade that closes the gap. Thus, we cannot solve $w$ for a given accretion rate. Thus, we can only illustrate by hand how $\rho(s)$ and $E_{\|}(s)$ are related in figure 3. A vacuum gap can be naturally formed around the null surface, because $d E_{\|} / d s$ changes sign at the null surface. There remains, however, another problem how to supply the charges that emit photons and how to realize a force-free magnetosphere outside the gap. This motivates us to consider pair creation inside and outside the gap.

\subsubsection{Non-vacuum gap around the null surface}

To pursue the supply of charges into the magnetosphere, we next consider a non-vacuum gap, $0<j \leq 1$. In a non-vacuum gap, $\rho_{\mathrm{GJ}}$ is partly canceled by $\rho$, leading to a smaller $\left|E_{\|}\right|$than the vacuum value. To grasp the relationship between $\rho(s)$ and $E_{\|}(s)$, we only show the representative results of their distribution in this section, leaving the further details of electrodynamics in $\oint 5$.

We solve the gap by the method described in $\S[5$ for 
the $\mathrm{BH}$ magnetosphere of IC 310, adopting $j=0.5$ and and dimensionless accretion rate $\dot{m}=3.16 \times 10^{-5}$, where

$$
\dot{m} \equiv \frac{\dot{M}}{\dot{M}_{\mathrm{Edd}}}
$$

$\dot{M}$ denotes the mass accretion rate, $\dot{M}_{\text {Edd }}$ the Eddington accretion rate

$$
\dot{M}_{\text {Edd }} \equiv \frac{L_{\text {Edd }}}{\eta_{\text {eff }} c^{2}}=1.39 \times 10^{27} M_{9} \mathrm{~g} \mathrm{~s}^{-1},
$$

$L_{\text {Edd }}$ the Eddington luminosity, and the conversion efficiency is assumed to be $\eta_{\mathrm{eff}}=0.1$. The resultant $\rho(s)$ and $E_{\|}(s)$ distribution is presented in figure 4, The gap inner and outer boundaries are located at $s_{1}=-0.201 r_{\mathrm{g}}$ and $s_{2}=2.345 r_{\mathrm{g}}$, respectively. Quantities $\rho / B, \rho_{\mathrm{GJ}} / B$, and $E_{\|}$are depicted only within the gap, $s_{1}<s<s_{2}$. The maximum $E_{\|}$becomes $6.19 \times 10^{1}$ statvolt $\mathrm{cm}^{-1}$. Because of the screening by the discharged pairs, this value is about half of the vacuum-case (i.e., $j=0$ case) value $E_{\|}(0)=1.35 \times 10^{2}$ statvolt $\mathrm{cm}^{-1}$ for the same $w / 2=s_{2}$ (eq. [12). The potential drop becomes $V_{\text {gap }}=4.34 \times 10^{14}$ statvolt for the $j=0.5$ case.

We also present the case of the marginally superGJ case, $j=1$, in figure [5] Since $\rho=\rho_{\text {GJ }}$ holes at the outer boundary, $d E_{\|} / d s$ vanishes there, requiring no surface charge. The maximum $E_{\|}$becomes $2.08 \times 10^{1}$ statvolt $\mathrm{cm}^{-1}$, and the potential drop becomes $V_{\text {gap }}=1.72 \times 10^{14} \mathrm{~V}$. Note that the total luminosity of the gap is given by the product of $V_{\text {gap }}$ and the current flowing through the gap, the latter of which is proportional to $j$. Since the product $j V_{\text {gap }}$ decreases only $20 \%$ from $j=0.5$ to $j=1$, we find that the gap luminosity depends on $j$ relatively weakly, as long as $0.5 \leq j \leq 1$. We thus adopt $j=1$ as the representative value in the present paper.

Figures 4 and 5 show that the gap width $w=s_{2}-s_{1}$ slightly increases from $j=0.5$ to $j=1.0$. This is because the weaker $E_{\|}$for $j=1$ results in less energetic IC photons, which require longer mean-free path to materialize as pairs, thereby increasing $w$. However, at the same time, the doubled particle flux of the $j=1$ case (from the $j=0.5$ case) results in an increased IC photon flux, which in turn contribute to decrease $w$ (for details, see Hirotani (2013)). Because of such negative feedback effects, gap solution exists in a vast expanse of parameter space in BH and pulsar magnetospheres. For this reason, classic pulsar vacuum outer-gap model works qualitatively well, because the errors incurred by the vacuum assumption and the GJ charge density in the gap partly cancel each other and keep the conclusions more or less close to the non-vacuum gap model.

The maximum current density flowing through the gap, is limited by $c\left|\rho_{\mathrm{GJ}}\left(s=s_{2}\right)\right|$. Thus, the wider the gap extends, the greater $c\left|\rho_{\mathrm{GJ}}\left(s_{2}\right)\right|$ becomes. For example, near the null surface, we can expand $\rho_{\mathrm{GJ}}$ to obtain

$$
c\left|\rho_{\mathrm{GJ}}\left(s_{2}\right)\right| \approx c\left|\rho_{\mathrm{GJ}}{ }^{\prime}\right| s_{2} \text {. }
$$

Integrating over the hemisphere of the horizon, we obtain the total current flowing in the entire magnetosphere,

$$
J_{\max } \approx\left(c\left|\rho_{\mathrm{GJ}}{ }^{\prime}\right| s_{2}\right) \cdot 2 \pi r_{\mathrm{H}}^{2} \approx J_{\mathrm{tot}} \frac{s_{2}}{r_{\mathrm{H}}},
$$

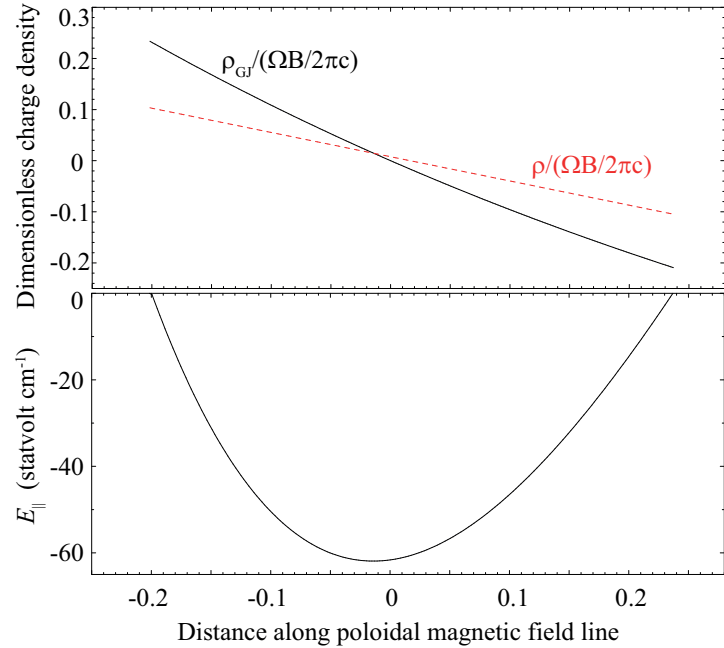

FIG. 4.- Charge densities and acceleration electric field in a non-vacuum gap with mild screening, $j=0.5$. Both the inner and outer boundaries are solved as a free boundary problem for the dimensionless accretion rate, $\dot{m}=3.16 \times 10^{-5}$ (for the detailed methods, see $\S$ 5). Top panel: Charge density per magnetic flux tube (red dashed curve), and the Goldreich-Julian charge density per magnetic flux tube (black solid curve) as a function of the distance $s$ from the null surface. Bottom panel: Magnetic-fieldaligned electric field.

where

$$
J_{\text {tot }} \equiv \frac{\left(\omega_{\mathrm{H}}-\Omega_{\mathrm{F}}\right) B}{2 \pi} \cdot 2 \pi r_{\mathrm{H}}^{2}=\left(\omega_{\mathrm{H}}-\Omega_{\mathrm{F}}\right) B r_{\mathrm{H}}^{2}
$$

represent the maximally possible electric current flowing along the magnetic field lines threading the horizon. The $\omega_{\mathrm{H}}-\Omega_{\mathrm{F}}$ factor in $J_{\text {tot }}$ comes from the fact that $\rho_{\mathrm{GJ}}$ is proportional to $\omega_{\mathrm{H}}-\Omega_{\mathrm{F}}$ at the horizon.

Combining equations (13) and (19), we obtain the maximally possible luminosity of the gap,

$$
L_{\text {gap } \text { max }}=V_{\text {gap }} J_{\text {max }} \approx(\mathrm{EMF}) \cdot J_{\text {tot }}\left(\frac{s_{2}}{r_{\mathrm{H}}}\right)^{4}=L_{\mathrm{sd}}\left(\frac{s_{2}}{r_{\mathrm{H}}}\right)^{4} \text {. }
$$

If $E_{\|}$took the vacuum value (i.e., eq. [12]) but the current was $J_{\max }$, which would contradict each other, the gap luminosity would become $L_{\text {gap,max }}$. In a non-vacuum gap, $E_{\|}$is less than the vacuum value and the current is less than $J_{\text {max }}$; thus, the gap luminosity should be less than equation (21). The $s_{2}{ }^{4} \approx(w / 2)^{4}$ dependence comes from the fact that $E_{\|}$is proportional to $s_{2}{ }^{2}$, that the potential drop is proportional to $E_{\|} s_{2}$, and that the maximum current is also proportional to $s_{2}$ (eq. 19]).

In short, a non-vacuum gap can be formed around the null surface, because $\rho-\rho_{\mathrm{GJ}}$, and hence $d E_{\|} / d s$ naturally changes sign within the gap, as demonstrated in figures 4 and 5 .

\subsubsection{Gap formation away from the null surface}

Let us next examine the possibility of the formation of a gap away from the null surface, e.g., around the stagnation surface. In figure 6, we sketch the possibility of the formation of a gap around $s \approx 1.57 \mathrm{r}_{\mathrm{g}}$. If there is no particle injection across the boundaries, only electrons exist at the outer boundary, and only positrons at the inner boundary (top panel). In this case, $\rho-\rho_{\mathrm{GJ}}$ is positive definite (middle panel), resulting in a monotonically increasing $E_{\|}$in the gap (bottom panel). Thus, the inner 


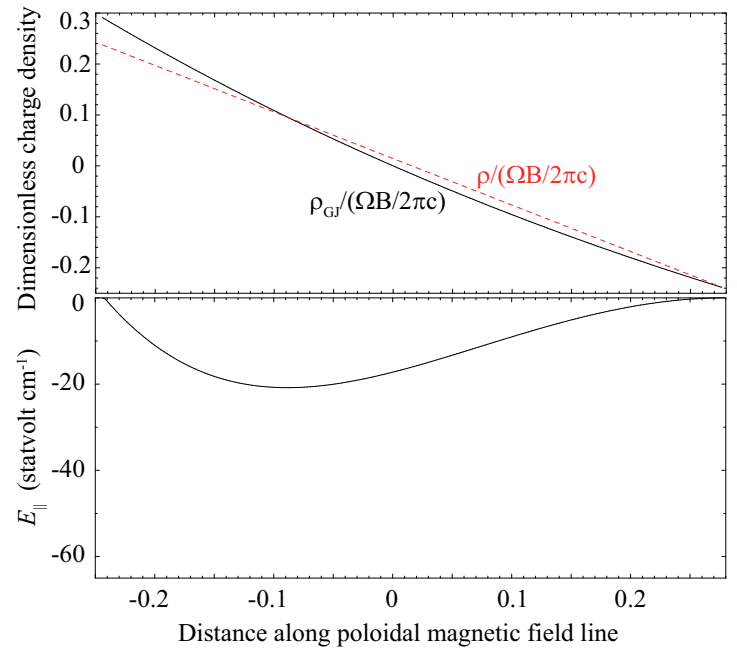

FIG. 5.- Similar figure as figure 4 but with the strongest screening, $j=1.0$. Other parameters than $j$ are the same as figure 4 Because of less efficient gamma-ray emission, the gap width enlarges compared to the $j=0.5$ case (fig. 4).

boundary cannot be formed in this case if we set $E_{\|}=0$ at the outer boundary.

To change the sign of $\rho-\rho_{\mathrm{GJ}}$, we must consider the injection of electrons across the inner boundary, in the same way as in pulsar outer gap model (Hirotani \& Shibata 2001). It is possible if a smallamplitude, residual $E_{\|}$separate the charges at $s<s_{1}$, and if a fraction of the returned electrons enter the gap. In this case, the position of the gap center is determined solely by the injected current across the boundary. Thus, if the injected current density per magnetic flux tube, $c e\left(n_{-} / B\right)_{1}$, coincidentally matches $c \rho_{\mathrm{GJ}} / B$ at the stagnation surface, the gap appears around the stagnation surface, where the subscript 1 shows that the quantity is evaluated at the inner boundary. Because $\rho-\rho_{\mathrm{GJ}}$ changes sign (middle panel of fig. (7), $E_{\|}$also changes sign (bottom panel) to close the gap. We interpret that the treatment of LR11 and BT15 corresponds to this case, although it is not explicitly mentioned in their papers.

In short, the position of a gap shifts outwards (or inwards) if there is a leptonic current injection across the inner (or outer) boundary. The center of the gap is determined by the strength of the injected current. Only when the injected current density $c e\left(n_{-} / B\right)$ at the inner boundary matches $c \rho_{\mathrm{GJ}} / B$ at the stagnation surface, the gap center can be located at the stagnation surface.

In $\mathrm{BH}$ gap models, there have been two main ideas about the position of the gap. One considers gaps at the null surface (Beskin et al. 1992, HO98), and the other at the separation surface (LR11,BT15). In this paper, we pursue the first possibility, because there is no essential difference in gap electrodynamics between the two cases, and because no current injection may be the simplest assumption. For instance, the gap closure condition (§ 4.5) is much simplified in the former case, because we do not have to consider additional $\gamma$-ray emission by the injected charges.

\section{RADIATIVELY INEFFICIENT ACCRETION}

In this paper, we consider a situation in which plasma accretion takes place in the lower latitudes, that is, near the rotational equator with a certain vertical thick-

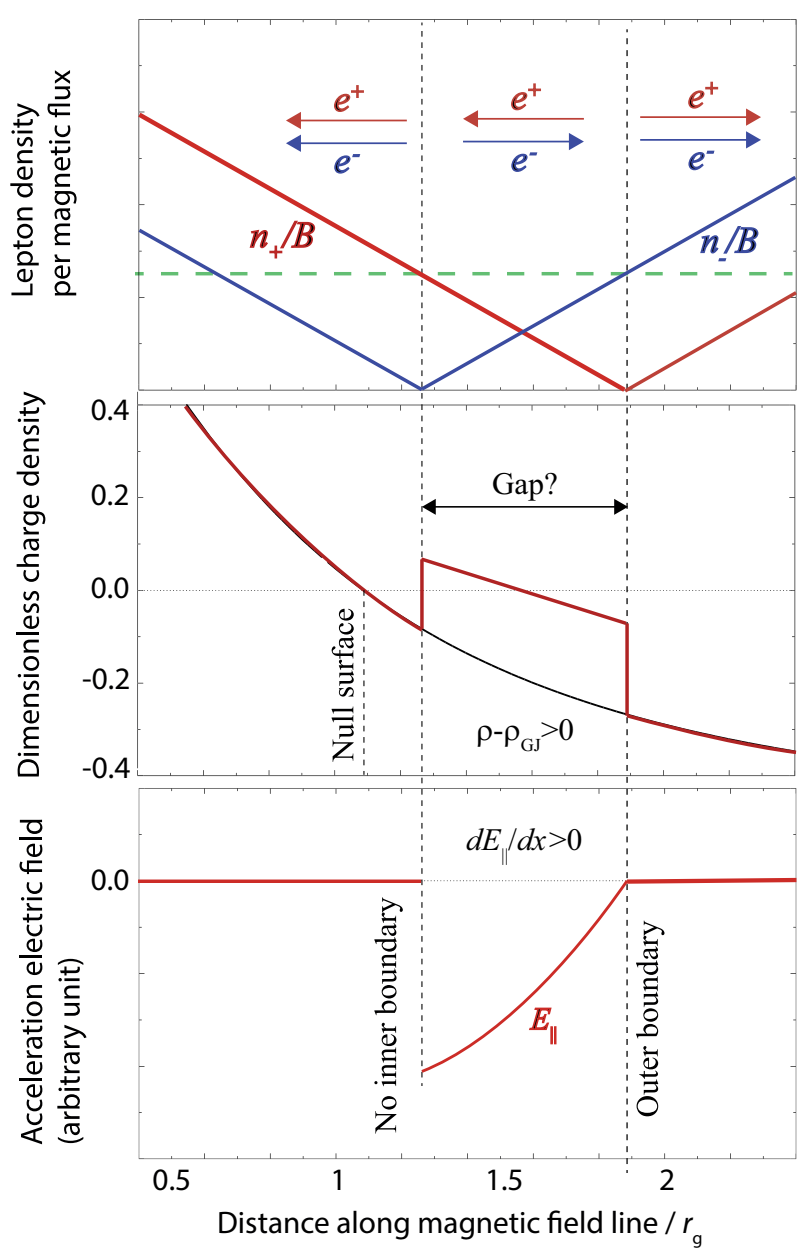

FIG. 6.- Similar figure as figure 4 but the gap position is artificially shifted from the null surface. In the shifted gap, a single-signed magnetic-field-aligned electric field, $E_{\|}$, accelerates electrons and positrons in the opposite directions.

ness (Krolik et al. 2005; McKinnev et al. 2006, 2007a, b; Punsly et al. 2009; (Punsly 2011). Such an accretion cannot penetrate into the higher latitudes, that is, in the polar funnel, because the centrifugal-force barrier prevents plasma accretion towards the rotation axis, and because the time scale for magnetic Rayleigh-Taylor instability or turbulent diffusion is long compared to the dynamical time scale of accretion. In this evacuated funnel, the poloidal magnetic field lines resemble a split monopole in a time-averaged sense (Hirose et al. 2004; McKinnev et al. 2012). The lower-latitude accretion emits $\mathrm{MeV}$ photons into the higher latitudes, supplying electron-positron pairs in the funnel. If the pair density in the funnel becomes less than the GJ number density, an electric field arises along the magnetic field. Thus, a plasma accretion in the lower altitudes and a gap formation in the higher altitudes are compatible in a $\mathrm{BH}$ magnetosphere.

From multi-frequency radio observations, the jet power of IC 310 is estimated to be $L_{\text {jet }}=2 \times 10^{42} \mathrm{ergs} \mathrm{s}^{-1}$ (Sijbring \& de Bruyn 1998). If this jet is accretionpowered, we find that the dimensionless accretion rate will be

$$
\dot{m}=\eta_{\mathrm{j}}{ }^{-1} L_{\mathrm{jet}} / L_{\mathrm{Edd}}=1.6 \times 10^{-4}\left(\frac{\eta_{\mathrm{j}}}{0.1}\right)^{-1} M_{9}{ }^{-1},
$$




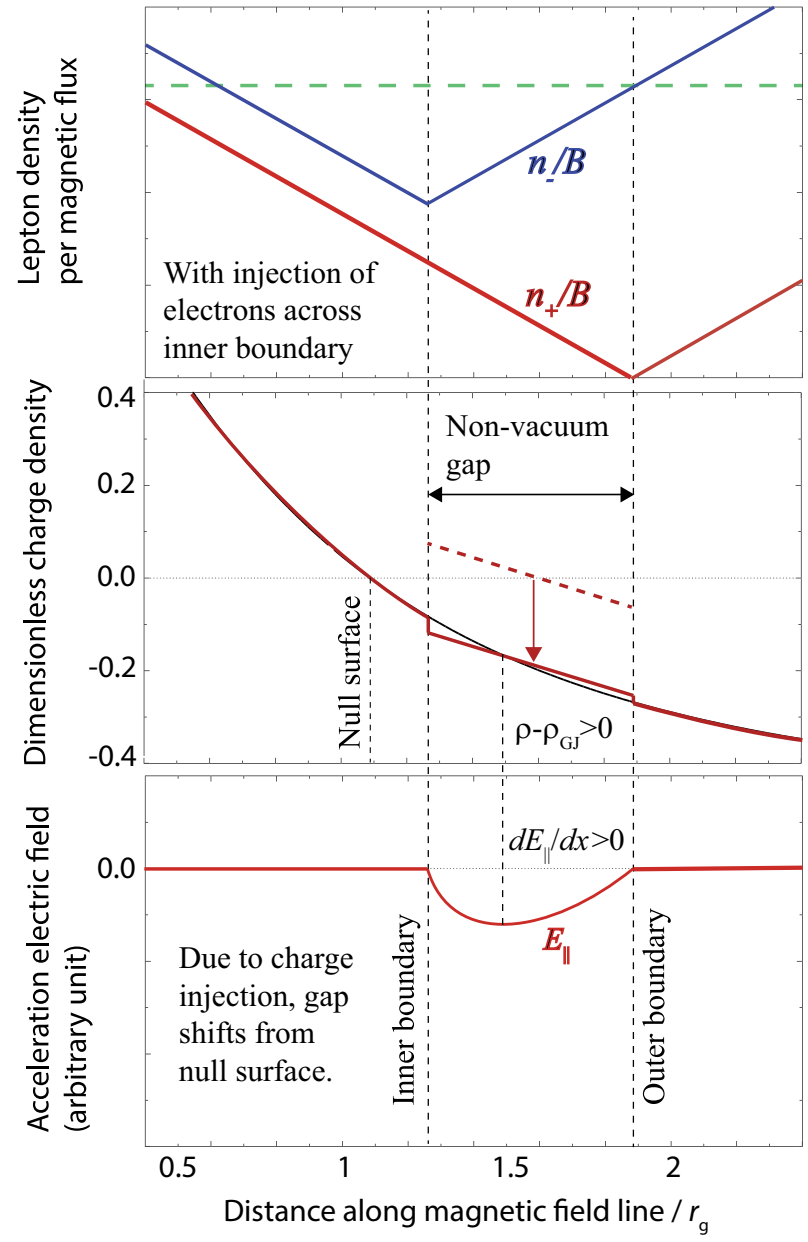

FIG. 7.- Similar figure as figure 4 but an electric current is injected across the inner boundary so that $\rho-\rho_{\mathrm{GJ}}$ may change sign within the gap.

where $\eta_{\mathrm{j}}$ denotes the energy conversion efficiency from accretion into jet (McKinnev et al. 2012). Thus, we can evaluate $\dot{m} \sim 10^{-4}$ or slightly greater, depending on the value of $\eta_{\mathrm{j}}$.

If the jet is BH-rotation-powered, the BlandfordZnajek process energizes the jet. Its power can be estimated to be (Blandford \& Znajek 1977)

$$
L_{\mathrm{sd}} \approx 10^{42}\left(\frac{a}{M}\right)^{2}\left(\frac{M_{9}}{0.3}\right)^{2} B_{3}^{2} \operatorname{ergs~s}^{-1},
$$

where $B_{3}=B /\left(10^{3} \mathrm{G}\right)$. If the magnetic pressure is in equilibrium with the gas pressure, we obtain the equilibrium magnetic field strength (LR11)

$$
B_{\mathrm{eq}} \approx 4 \times 10^{4} \dot{m}^{1 / 2} M_{9}^{-1 / 2} \mathrm{G} .
$$

Equating $L_{\text {sd }}$ with $L_{\text {jet }}$, and setting $B \approx B_{\text {eq }}$, we obtain $B_{3} \approx 1.4$ and $\dot{m} \sim 4 \times 10^{-4}$.

Combining these two estimates, we can infer the accretion rate to be $\dot{m} \sim 2 \times 10^{-4}$ on time-averaged sense. Nevertheless, it is worth noting that this argument does not restrict the value of $\dot{m}$ on much shorter timescales than the jet propagation times scale at radio-emitting regions, the latter of which is much longer than the light crossing time of the horizon.

At such a low accretion rate, $\dot{m} \sim 10^{-4}$ or less, the accretion flow becomes radiatively inefficient (Ichimaru 1977; Naravan \& Yi 1994, 1995; Naravan et al. 1997; Nakamura et al. 1997). In the present paper, for analytical purpose, we adopt the self-similar solution of an advection-dominated accretion flow (ADAF) in Newtonian approximation (Mahadevan 1997), skipping to incorporate general relativistic corrections (Manmoto 2000; Li et al. 2008) into the ADAF model. Such plasma accretion takes place in the lower latitudes (i.e., near the rotational equator with a certain vertical thickness) and emit photons into many directions including the higher latitudes (i.e., the polar funnel) where jets are launched. Thus, to estimate the density of the created pairs in the higher latitudes (e.g., around the colatitude $\theta \sim 10^{\circ}$ or $20^{\circ}$ ), we must examine the flux of the $\mathrm{MeV}$ photons emitted by the ADAF.

To evaluate the flux of ADAF MeV photons, we consider the free-free emission in the inner region of the ADAF. The free-free emission spectrum peaks around $\nu_{\mathrm{p}}=k T_{\mathrm{e}} / h$, where $T_{\mathrm{e}}$ denotes the electron temperature, $k$ the Boltzmann constant, and $h$ the Planck constant. Near the peak, eq. (30) of Mahadevan (1997) gives the soft photon luminosity

$$
L_{\mathrm{s}}=2.06 \times 10^{38} T_{\mathrm{e}, 10} m \dot{m}^{2} \mathrm{ergs} \mathrm{s}^{-1}
$$

if the viscosity parameter is $\alpha=0.3, c_{1}=0.5$, $r_{\max } / r_{\min }=10^{3} / 3$, and $T_{\mathrm{e}, 10} \equiv T_{\mathrm{e}} /\left(10^{10} K\right)>0.5$ (see Mahadevan (1997) for details). Thus, around $r=6 r_{\mathrm{g}}$, the $\mathrm{MeV}$ photon number density becomes

$$
n_{\mathrm{s}}=4.72 \times 10^{11} M_{9}{ }^{-1} \dot{m}^{2}\left(\frac{r}{6 r_{\mathrm{g}}}\right)^{-2} \mathrm{~cm}^{-1} .
$$

Following the logic around equation (6) of LR11 we obtain the number density of pairs created by the collisions of these $\mathrm{MeV}$ photons,

$$
n_{ \pm}=8.88 \times 10^{12} M_{9}^{-1} \dot{m}^{4}\left(\frac{r}{6 r_{\mathrm{g}}}\right)^{-3} \mathrm{~cm}^{-1} .
$$

Evaluating $B$ with equation (24), we obtain the GJ number density,

$$
n_{\mathrm{GJ}}=4.41 \times 10^{-2} M_{9}{ }^{-3 / 2} \dot{m}^{1 / 2} \frac{a}{M} \frac{\Omega_{\mathrm{F}}}{\omega_{\mathrm{H}}} \mathrm{cm}^{-1},
$$

Thus, we obtain

$$
\frac{n_{ \pm}}{n_{\mathrm{GJ}}}=2.01 \times 10^{14} M_{9}^{1 / 2} \dot{m}^{7 / 2}\left(\frac{a}{M}\right)^{-1}\left(\frac{\Omega_{\mathrm{F}}}{\omega_{\mathrm{H}}}\right)^{-1}\left(\frac{r}{6 r_{\mathrm{g}}}\right)^{-3} .
$$

It follows that $n_{ \pm}<n_{\text {GJ }}$ approximately holds if $\dot{m}<$ $10^{-4}$ for $M_{9}=0.3$. In another word, the time-averaged value of $\dot{m} \sim 2 \times 10^{-4}$ gives an order of magnitude greater pair density than the GJ value. Therefore, for IC 310, we expect that the BH gap is quenched by the ADAFprovided pairs along any magnetic field lines during a substantial portion of time.

It is, however, noteworthy that $n_{ \pm} / n_{\mathrm{GJ}}$ strongly depends on $\dot{m}$. For instance, if $\dot{m}$ is halved, $n_{ \pm} / n_{\mathrm{GJ}}$ decreases one order of magnitude. Thus, it is worth considering the cases in which a smaller accretion rate, $\dot{m} \ll 10^{-4}$, is achieved in a limited space and time. For instance, because a lower-latitude accretion in a geometrically thick disk is known to be highly variable due to 
turbulence (Hirose et al. 2004; McKinney et al. 2007a), $\dot{m} \ll 10^{-4}$ may be achieved during a short period of time. What is more, it is suggested by three-dimensional MHD simulations that an accretion of magnetized plasmas tends to be highly variable near the horizon particularly when the BH spin approaches the extreme value, $a \approx 0.998 r_{\mathrm{g}}$ (Krolik et al. 2005). It was also analytically pointed out that a strongly magnetized plasma accretion tends to be highly variable near the horizon (Hirotani et al. 1993). This is because the meridional current suffers a large-amplitude fluctuation (compared to e.g., the radial magnetic field fluctuations) at the fastmagnetosonic surface, which is located in the vicinity of the horizon in a magnetically dominated magnetosphere (Phinney 1983). This results in a strong perturbation of the Lorentz force, leading to a large radial acceleration of the MHD inflow. This phenomena is solely general relativistic (GR) in the sense that the plasma inertia and the causality at the horizon are essential. Thus, it will not happen in non-GR trans-fast-magnetosonic flows.

Since our viewing angle of IC 310 is constrained to be between $10^{\circ}$ and $20^{\circ}$ with respect to the jet axis (Aleksic et al. 2014b), we assume that $\dot{m}<10^{-4}$ is achieved in the higher latitudes intermittently. In this case, the BH gap of IC 310 will be switched on with a small duty cycle (i.e., during a small fraction of time).

\section{GAP ELECTRODYNAMICS}

We formulate a stationary particle accelerator exerted around a rotating $\mathrm{BH}$ in this section.

\subsection{Saturated Lorentz factors}

If the curvature drag force balances $e E_{\|}$, the electron Lorentz factors are saturated at the curvature-limited value,

$$
\gamma_{\text {curv }} \equiv\left(\frac{3 \rho_{\mathrm{c}}^{2}}{2 e} E_{\|}\right)^{1 / 4}
$$

where $\rho_{\mathrm{c}}$ denotes the curvature radius of the threedimensional particle path, which becomes comparable to the curvature radius of the magnetic field lines in a rotating magnetosphere (Hirotani 2011). Although the split-monopole solution specifies not only the poloidal magnetic field components but also the toroidal (i.e., azimuthal) one, we set $\rho_{\mathrm{c}}$ as a free parameter in the present paper, because a time-dependence accretion onto a $\mathrm{BH}$ will have a spatially and temporary varying magnetic field near the horizon (Krolik et al. 2005).

If the IC drag force balances $e E_{\|}$, on the other hand, the saturated Lorentz factor, $\gamma_{\mathrm{IC}}$, should be implicitly solved from the balance

$$
\frac{1}{c} \int_{E_{\min }}^{\gamma_{\mathrm{IC}} m_{\mathrm{e}} c^{2}} E_{\gamma} d E_{\gamma} \int_{E_{\min }}^{E_{\max }} \frac{d \sigma}{d E_{\gamma}} \frac{d F_{\mathrm{s}}}{d E_{\mathrm{s}}} d E_{\mathrm{s}}=e E_{\|},
$$

where $m_{\mathrm{e}}$ denotes the rest mass of the electron, $E_{\gamma}$ and $E_{\mathrm{s}}$ the (emitted) hard-photon and (input) soft-photon energies, respectively, $E_{\min }$ and $E_{\max }$ the lower and upper bound energies we are considering, $d \sigma / d E_{\gamma}$ the Klein-Nishina differential cross section, $d F_{\mathrm{s}} / d E_{\mathrm{s}}$ the differential flux of the background soft photon field. Note that $d \sigma / d E_{\gamma}$ has $\gamma_{\mathrm{IC}}$ dependence. In $\S 5$, we demonstrate that the IC scatterings mainly take place in the extreme Klein-Nishina regime.
We assume that the input ADAF photon field (Mahadevan 1997) is isotropic in the BH rest frame. To cover the photon energies from radio wavelengths (of $\mathrm{ADAF}$ emission) to the $\mathrm{VHE}$, we adopt $E_{\min }=$ $5.11 \times 10^{-6} \mathrm{eV}$ and $E_{\max }=5.11 \times 10^{17} \mathrm{eV}$, dividing the energies logarithmically into 198 bins. To estimate the normalization of the ADAF photon differential flux at the gap, we divide the total ADAF differential luminosity (Mahadevan 1997) by $4 \pi\left(10 r_{\mathrm{g}}\right)^{2}$, assuming that the ADAF emission flux is homogeneous at $r<10 r_{\mathrm{g}}$ but reduces by the $\left(r / 10 r_{\mathrm{g}}\right)^{-2}$ factor at $r>10 r_{\mathrm{g}}$; this assumption is reasonable, because the sub-millimeter-IR photons, which most efficiently contribute for both IC scatterings and photon-photon pair creation, are emitted from the inner-most region of ADAF, $r \approx 10 r_{\mathrm{g}}$. We compute both photon-photon pair creation and IC scatterings, from the gap to a large enough Boyer-Lindquist radius, $r=60 r_{\mathrm{g}}$. If we evaluated the $\mathrm{ADAF}$ flux at $r=6 r_{\mathrm{g}}$ (instead of $10 r_{\mathrm{g}}$ ), the similar solutions would be obtained at smaller $\dot{m}$. Nevertheless, emission properties of the gap (e.g., spectrum near the critical accretion rate) little changes by the normalization of the ADAF photon flux. Note that the charge-starvation condition $n_{ \pm} / n_{\mathrm{GJ}}<1$ (eq. 29]) is more easily satisfied if the ADAF flux is normalized at $r=10 r_{\mathrm{g}}$ (than at $r=6 r_{\mathrm{g}}$ ). See also $\S 6.1 .2$ for the impact of the normalization of the soft photon field on the charge-starvation condition.

The actual Lorentz factor is computed by

$$
\gamma^{-1}=\gamma_{\mathrm{curv}^{-1}}+\gamma_{\mathrm{IC}}{ }^{-1}
$$

which is almost equivalent with $\gamma=\min \left(\gamma_{\text {curv }}, \gamma_{\mathrm{IC}}\right)$.

\subsection{Curvature process}

At a Lorentz factor $\gamma$, a single electron emits (Rybicki \& Lightman 1979)

$$
\left(P_{\nu}\right)_{\mathrm{cv}}=\frac{\sqrt{3} e^{2}}{h \nu} \frac{\gamma}{\rho_{\mathrm{c}}} F\left(\frac{\nu}{\nu_{\mathrm{c}}}\right)
$$

photons per unit frequency $\nu$ per unit time, where

$$
F(x) \equiv x \int_{x}^{\infty} K_{5 / 3}(\xi) d \xi
$$

$x \equiv \nu / \nu_{\mathrm{c}}$ and $K_{5 / 3}$ denotes the modified Bessel function of $5 / 3$ order. The characteristic frequency is defined as

$$
\nu_{\mathrm{c}} \equiv \frac{3}{4 \pi} \frac{\gamma^{3} c}{\rho_{\mathrm{c}}} .
$$

Particles have different Lorentz factors at different positions. We compute the evolution of $\gamma$ as a function of position, assuming that electrons are created homogeneously at each position. If pairs are created at $s=s_{\mathrm{cr}}$, the electrons are accelerated outward to attain the Lorentz factor at $s$,

$$
\gamma(s)=\min \left(\frac{-e}{m_{\mathrm{e}} c^{2}} \int_{s_{\mathrm{cr}}}^{s} E_{\|}(s) d s, \gamma_{\mathrm{curv}}\right) .
$$

To count up the photons emitted from various positions, we divide the full gap width into $n_{x}$ parts along the particle path. Accordingly, we can compute the number of photons emitted outwardly in the $i$-th energy bin 
per unit time by

$$
\left(N_{\mathrm{cv}}\right)_{i}=\frac{N_{\mathrm{e}}}{n_{x}} \int_{\nu_{i-1}}^{\nu_{i}}\left(P_{\nu}\right)_{\mathrm{cv}} d \nu
$$

where $N_{\mathrm{e}}$ denotes the total number of electrons existing in the entire gap, $\nu_{i-1}$ and $\nu_{i}$ the lower and upper bound of the $i$-th photon energy bin. In $\S 4.4$, we will describe how $N_{\mathrm{e}}$ is computed.

\subsection{Inverse-Compton process}

Next, we consider the emission properties of the IC process. At a Lorentz factor $\gamma$, a single electron emits

$$
\left(P_{\nu}\right)_{\mathrm{IC}}=\frac{3 \sigma_{\mathrm{T}}}{16}\left(1-\beta \mu_{\mathrm{c}}\right) \int_{0}^{\infty} d E_{\mathrm{s}} \frac{d F_{\mathrm{s}}}{d E_{\mathrm{s}}} \int_{-1}^{1} d x^{*} \int_{0}^{2 \pi} d \alpha^{*} f_{\mathrm{IC}}
$$

photons per unit frequency per unit time, where $f_{\mathrm{IC}}=$ $f_{\mathrm{IC}}\left(x^{*}, \alpha^{*}, E_{\mathrm{s}}, \mu_{\mathrm{c}}, \gamma\right)$ is defined by equations $(26)-(28)$ of Hirotani et al. (2003), and $\mu_{\mathrm{c}}$ denotes the cosine of the collision angle. Assuming isotropic IC scatterings in the $\mathrm{BH}$ rest frame when we average over the collision angles, we obtain the number of photons emitted outwardly in the $i$-th energy bin per unit time as follows:

$$
\left(N_{\mathrm{IC}}\right)_{i}=\frac{N_{\mathrm{e}}}{n_{x}} \int_{\nu_{i-1}}^{\nu_{i}} d \nu \frac{1}{2} \int_{-1}^{1}\left(P_{\nu}\right)_{\mathrm{IC}} d \mu_{\mathrm{c}}
$$

\subsection{Photon-photon pair creation}

The $\gamma$-rays emitted via curvature or IC process, are partly absorbed by colliding with the ADAF soft photons. The absorption optical depth for the photons in the $i$-th energy bin becomes

$$
\tau_{i}=\frac{s_{2}-s_{1}}{c} \cdot \frac{1}{2} \int_{-1}^{1} d \mu_{\mathrm{c}} \int_{E_{\mathrm{th}}}^{\infty} \sigma_{\mathrm{p}}\left(E_{i}, E_{\mathrm{s}}, \mu_{\mathrm{c}}\right) \frac{d F_{\mathrm{s}}}{d E_{\mathrm{s}}} d E_{\mathrm{s}}
$$

where $\sigma_{\mathrm{p}}$ refers to the photon-photon pair creation meanfree path (Jauch \& Rohrlich 1955), $E_{i}=h\left(\nu_{i-1}+\nu_{i}\right) / 2$ denotes the $\gamma$-ray energy. The threshold energy is given by $E_{\mathrm{th}}=\left[2 /\left(1-\mu_{\mathrm{c}}\right)\right]\left(m_{\mathrm{e}} c^{2}\right)^{2} / E_{i}$. The photon-photon collision is assumed to be isotropic. Since the typical energy of the curvature photons is around $\mathrm{TeV}$, only the ADAF photons above near-IR frequency contribute for pair creation. However, at $\dot{m}<10^{-4}$, the number flux of these photons is typically 8 orders of magnitude smaller than the sub-millimeter-IR photons, which will effectively collide with $\sim 100 \mathrm{TeV}$ IC photons. Thus, for low-luminosity active galactic nuclei (AGNs), curvature photons do not effectively materialize as pairs in the $\mathrm{BH}$ gap, forming a striking contrast to pulsar gaps. This point will be discussed in $\S 5$ in detail.

\subsection{Gap closure condition}

Although the curvature photons do not materialize in a $\mathrm{BH}$ gap of a low-luminosity AGN, we consider both the curvature and IC processes for completeness. The number of pairs cascaded from a single electron is given by

$$
\mathcal{M}=\Sigma_{i}\left[\left(N_{\mathrm{cv}}\right)_{i}+\left(N_{\mathrm{IC}}\right)_{i}\right]\left[1-\exp \left(-\tau_{i}\right)\right],
$$

where $i$ denotes the photon energy bin. For simplicity, we assume that the same $\mathcal{M}$ can be applied for both the outgoing electrons and the ingoing positrons. For a gap to be sustained stationarily, multiplicity $\mathcal{M}$ should be unity. That is, a single electron materializes into $\mathcal{M}$ pairs on average within the gap. The returned $\mathcal{M}$ positrons emit copious $\gamma$-rays inwards, $\mathcal{M}^{2}$ of which materialize as pairs. As a result, a single electron cascades into $\mathcal{M}^{2}$ electrons after one 'round trip'. We thus obtain $\mathcal{M}=1$ as the condition for a gap to be sustained stationarily. If this inward-outward symmetry breaks down, we must impose a more complicated gap closure condition that the product of the inward and outward multiplicities should become unity (Hirotani 2013).

From the condition of $\mathcal{M}=1$, we can update $s_{2}$. We solve equation (9) from $s=s_{2}$ to the inner boundary $s=s_{1}$ where $E_{\|}$vanishes. The inner and outer boundaries, $s=s_{1}$ and $s=s_{2}$, are determined as a freeboundary problem, because we impose the gap closure condition, $\mathcal{M}=\infty$ and $d E_{\|} / d s$ at $s=s_{2}$ by specifying $j$ through equations (9) and (10). The updated $E_{\|}(s)$ is then substituted into equations (30) and (31) to update $\gamma(s)$. Subsequently, $\left(N_{\mathrm{CV}}\right)_{i}$ and $\left(N_{\mathrm{IC}}\right)_{i}$ are updated by (33), (35), (37), (38), and (39). Then $\mathcal{M}=1$ (eq. 41) updates $s_{2}$ again. We iterate this process until quantities converge.

\section{APPLICATION TO IC 310}

We apply the method described above to the radio galaxy IC 310. This nearby active galaxy $(z=0.0189)$ has been detected with Fermi/LAT (Neronov et al. 2010) in high energy gamma-rays and with MAGIC (Aleksic et al. 2011, 2014b) in VHE. Also in X-rays, non-thermal point-like emissions have been detected (Schwarz et al. 1992; Rhee, Burns \& Kowalsky 1994; Sato et al. 2005).

Using the $M-\sigma$ relation, one can estimate the mass of the central black hole of IC 310 to be $(2.4 \pm 0.5) \times 10^{8} M_{\odot}$ from the velocity dispersion of $(229.6 \pm 5.9) \mathrm{km} \mathrm{s}^{-1}$ in the host galaxy (McElrov 1995; Simien \& Prugniel 2002). On the other hand, using the fundamental plane of black hole activity (Merloni. Heinz \& di Matteo 2003), one obtains a higher mass $\sim 4 \times 10^{8} M_{\odot}$ from the $2-10 \mathrm{keV}$ X-ray flux of $6.4 \times 10^{-12} \mathrm{erg} \mathrm{cm}^{-2} \mathrm{~s}^{-1}$ (Eisenacher et al. 2013) and the 1.7 and $8.4 \mathrm{GHz}$ radio flux density of 0.1 Jy (Schulz, Kadler \& Ros et al. 2015). Thus, we adopt $M=0.3 M_{9}$ as the mass of the central $\mathrm{BH}$ of IC 310. See also Aleksic et al. (2014b) for details.

The split-monopole solution specifies both the poloidal and toroidal components of the magnetic field (Michel 1973). However, in the present paper, we treat the magnetic curvature radius, $\rho_{\mathrm{c}}$, as a free parameter to examine the impact of instantaneous kinks and bends of local magnetic field ( $(5.8)$. As a fiducial value, we adopt $\rho_{\mathrm{c}}=r$, because $r$ gives an estimate of the typical curvature radius of a toroidally wound magnetic field line.

Furthermore, we adopt $j=1$ in equation (10). In general, the created current can be determined if we solve the position-dependent pair production from the set of Boltzmann equations of $e^{ \pm}$'s and photons. In addition, the value of $j$ should be consistent with the global flow pattern, which may be constrained by force-free simulations (Spitkovski 2006). Nevertheless, it is out of the scope of the present paper to pursue the appropriate values of $j$ by such arguments. Thus, in what follows, we 
simply parameterize $\rho / B$, adopting $j=1$.

\subsection{The case of equilibrium magnetic field}

Let us first evaluate the magnetic field strength with equation (24). Substituting $\dot{m}=10^{-4}$ and $M_{9}=0.3$ into equation (24), we find the equilibrium magnetic field strength, $B=B_{\text {eq }}=7.3 \times 10^{2} \mathrm{G}$. Thus, equation (23) gives $L_{\mathrm{sd}} \approx 5.3 \times 10^{41} \mathrm{ergs} \mathrm{s}^{-1}$. We should notice here that the Blandford-Znajek power, $L_{\mathrm{sd}}$, gives the upper limit of the gap luminosity, because the $\mathrm{BH}$ gap is only liberating a part of the rotational energy of the black hole. Near the black hole, the magnetic field tends to be radial (or split-monopole-like) due to the inertia of the plasmas and the causality at the horizon (Hirotani et al. 1992; McKinney et al. 2012). If we sum up the outward emission along such (quasi) radial field lines over the entire null surface, the resultant photon flux will become more or less isotropic. In this case, the photon flux to be detected little depends on the beaming angles of the emitted photons.

In contrast to $L_{\text {gap }}<5.3 \times 10^{41} \mathrm{ergs} \mathrm{s}^{-1}$ mentioned just above, MAGIC detected the isotropic luminosity of $\sim 2 \times 10^{44} \mathrm{ergs} \mathrm{s}^{-1}$ during the flare (Aleksic et al. 2014b). Thus, we must conclude that the BH gap can reproduce at most $0.3 \%$ of the observed flare luminosity of IC 310 if $B \leq B_{\text {eq. }}$. Since the gap will be quenched by the ADAF-provided pairs at $\dot{m}>10^{-4}$, there is no hope to obtain such a large gap luminosity as $L_{\text {gap }} \approx 2 \times 10^{44} \mathrm{ergs} \mathrm{s}^{-1}$ (eq. 211), if we adopted the equilibrium magnetic field for IC 310 .

\subsection{Possibility of stronger magnetic field around extreme Kerr hole}

To explore the possibility of a stronger $B$, let us consider a magnetized plasma around an extremely rotating $\mathrm{BH}, r_{\mathrm{g}}>a \geq 0.998 r_{\mathrm{g}}$ (Bardeen 1970; Thorne 1974). If a black hole is extremely rotating, causality does not allow an additional accretion of plasmas that have positive angular momenta, thereby halting accretion at the event horizon. As a result of this cancellation of the gravity by the centrifugal force, a strong magnetic field can be confined by the accumulated, dense plasmas. For instance, magnetohydrodynamic accretions have a vanishing Alfvenic Mach number, $M_{\mathrm{A}} \propto B n^{-1 / 2}$ Camenzind 1986a $\mathrm{b}$ ), and hence a diverging plasma density $n$, at the event horizon in the limit of $a \rightarrow r_{\mathrm{g}}$ (Hirotani et al. 1992). In addition, GR MHD simulation shows that the magnetic energy becomes approximately 30 times (or 100 times) greater for $a=0.998 r_{\text {g }}$ near the horizon, comparing with $a=0.9 r_{\mathrm{g}}$ (or $a=0$ ) case under similar initial and boundary conditions (Hirose et al. 2004). This enhancement of the magnetic field is due to a plasma compilation near the horizon. The enhanced magnetic field enters well within the ergosphere, having a non-negligible radial component in the funnel. Thus, in the higher latitudes, it is capable of extracting the hole's rotational energy efficiently.

To set up the model as simple as possible, we therefore assume $B \approx 10^{4} \mathrm{G}$ irrespective of $\dot{m}$ under $r_{\mathrm{g}}>$ $a \geq 0.998 r_{\mathrm{g}}$ in this paper. Although equation (23) gives $L_{\mathrm{sd}} \approx 10^{44} \mathrm{ergs} \mathrm{s}^{-1}$ in this case, which greatly exceeds the observed jet power by 50 times, we expect that such a strong magnetic field, $B>14 B_{\text {eq }}$, can be sustained near the horizon only in a much shorter period than the jet propagation time scale. In another word, the $\mathrm{BH}$ gap of IC 310 is activated only intermittently with a small duty cycle, e.g., $\sim 1 / 50=0.02$. Although it is important to confirm the feasibility of such a strong $B$ around an extremely rotating $\mathrm{BH}$ by a numerical analysis, such an investigation is irrelevant to the main subject of the present paper. If $B$ greatly exceeded $10^{4} \mathrm{G}$, on the other hand, the BHs energy and angular momentum would be electromagnetically extracted so efficiently that the extracted power would exceed the jet power of typical AGNs (Ghisellini et al. 2014). On these grounds, we assume that the $\mathrm{BH}$ is extremely rotating, $a=0.998 \mathrm{r}_{\mathrm{g}}$, and that $B \approx 10^{4} \mathrm{G}$ holds during the flare. For such an extreme Kerr $\mathrm{BH}$, the horizon radius $r_{\mathrm{H}}$ becomes about a half of the Schwarzshild radius.

\subsection{Gap structure versus accretion rate}

If the $\mathrm{BH}$ is extremely rotating at $a \approx 0.998 r_{\mathrm{g}}$, the null surface is located at radius $r=r_{0} \approx 2.1 r_{\mathrm{H}}$ in $0 \leq \theta<$ $\pi / 2$ (fig. 1) if $\Omega_{\mathrm{F}} \approx 0.3 \omega_{\mathrm{H}}$, irrespective of the field line geometry. In what follows, unless explicitly mentioned, we adopt $\theta=15^{\circ}$ (Aleksic et al. 2014b), and assume a split-monopole magnetic field (left panel of fig. 11). Note that the argument is subject to change only mildly if we adopt different field line geometry such as parabolic one (right panel of fig. 1), particularly at higher latitudes, $\theta<30^{\circ}$.

The full gap width $w=s_{2}-s_{1}$ increases with decreasing $\dot{m}$ as depicted in the top panel of figure 8 . If the accretion rate decreases to $4.87 \times 10^{-6}, r_{0}+s_{1} \rightarrow r_{\mathrm{H}}=1.06 r_{\mathrm{g}}$, that is, the inner boundary eventually touches down the horizon. Below this critical accretion rate, no gap solution exists.

In the middle panel, we present $\gamma_{\text {curv }}$ (eq. 30), $\gamma_{\mathrm{IC}}$ (eq. 31), and $\gamma$ (eq. 32) with dashed, thick dotted, and solid curves. Note that the IC drag force is explicitly computed by fully taking account of the Klein-Nishina effect. For reference, we also plot the IC-limited Lorentz factor $\gamma_{\text {Thomson }}$ that would be obtained if the scatterings take place in the Thomson regime with thin dotted curve. It is clear that the IC process takes place in the extreme Klein-Nishina regime and that the deviation from $\gamma_{\text {Thomson }}$ becomes prominent at $\dot{m} \ll 10^{-4}$. Since we compute $\gamma_{\text {Thomson }}$ using a single parameter in the same way as LR11 for reference purpose (instead of replacing the Klein-Nishina cross section with the Thomson cross section in equation 31), the thick and thin dotted curves do not match around $\dot{m} \sim 10^{-4}$. It also shows that the Lorentz factors are limited by the curvature process (eq. 30 ) in almost the entire range of $\dot{m}<10^{-4}$. Since the curvature drag force dominates the IC one, the gap luminosity is dominated by the curvature process. Nevertheless, explicit computation of the IC emission with the Klein-Nishina cross section is, indeed, essential. This is because the closure condition, $\mathcal{M}=1$, is satisfied by the IC photons, or equivalently because the curvature photons do not materialize as pairs colliding with ADAF soft photons. In short, the Lorentz factors are limited by $\gamma_{\text {curv }}$ in the entire range of $\dot{m}<10^{-4}$.

In the bottom panel, we plot the number density of the pairs created outside the gap via photon-photon collisions. Since a force-free magnetosphere can be sustained 


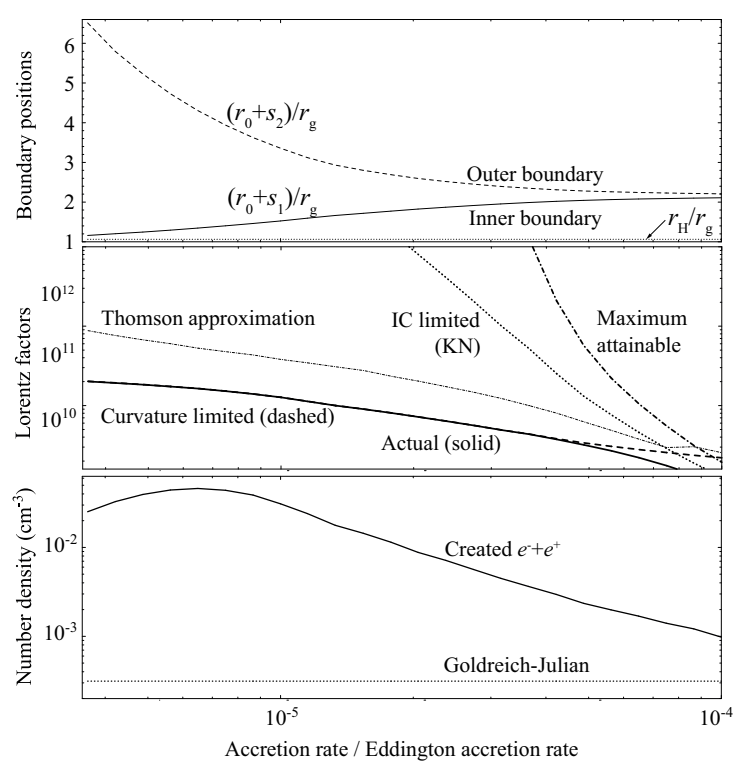

FIG. 8.- Quantities as a function of the dimensionless accretion rate $\dot{m}$. Top panel: Boundary positions in Boyer-Lindquist radial coordinate in $r_{\mathrm{g}}$ unit. The solid and dashed curves show the dimensionless positions of the inner and outer boundaries, $\left(r_{0}+s_{1}\right) / r_{\mathrm{g}}$ and $\left(r_{0}+s_{2}\right) / r_{\mathrm{g}}$. The dotted line represents the horizon position, $r_{\mathrm{H}} / r_{\mathrm{g}}=1.06$, which gives the physical lower limit of $\left(r_{0}+s_{1}\right) / r_{\mathrm{g}}$ (solid curve). Middle panel: The dash-dotted line shows the maximum attainable Lorentz factor computed from the potential drop of the non-vacuum gap. The thick dashed and dotted lines do the Lorentz factors limited by the curvature radiation and inverseCompton scatterings, respectively. The actual Lorentz factor of the accelerated electrons becomes as the solid line. For comparison, we also plot the Lorentz factors that would be obtained if the IC scatterings took place in the Thomson regime, as the thin dashed curve. Bottom panel: The solid line shows the density of the created electrons and positrons outside the gap, while the dashed one does the Goldreich-Julian value.

when the created pair density exceeds the GJ value, the present solutions give natural stationary solutions of the gap. The created pair density exceeds the GJ value more than two order of magnitude when the gap becomes most luminous, because $\sim 10^{4} \mathrm{TeV}$ IC photons cascade into $10^{2} \mathrm{TeV}$ pairs. Since the charge density becomes comparable to the GJ value in the gap when $w \gg r_{\mathrm{g}}$, it means that the multiplicity for a primary lepton to cascade into secondaries (and higher generation pairs) is also $\sim 10^{2}$. Since we are assuming $B=10^{4} \mathrm{G}$ irrespective of $\dot{m}$, the GJ density (horizontal dotted line) is constant with $\dot{m}$ in the present treatment.

\subsection{Gap distribution}

Let us examine the spatial distribution of the gap. Since the ADAF MeV photon density will be more or less homogeneous, we apply $\dot{m}=3.16 \times 10^{-5}$ for all the field lines. For illustration purpose, we adopt $\Omega_{\mathrm{F}}=0.3 \omega_{\mathrm{H}}$ for all the field lines as a practical compromise. It is, however, noteworthy that $-0.1 \Omega_{\mathrm{H}}<\Omega_{\mathrm{F}}<0.35 \Omega_{\mathrm{H}}$ is indicated in the funnel by the numerical simulations and the supporting analytical work both of which are claimed to represent radio loud AGN (McKinney et al. 2012; Beskin \& Zheltoukhov 2013).

At $\dot{m}=3.16 \times 10^{-5}$, the gap distributes on the meridional plane as depicted by the green region in figure 9 At $\theta=15^{\circ}, 45^{\circ}$, and $75^{\circ}$, the full gap thickness (along the magnetic field line on the poloidal plane) become $w=s_{2}-s_{1}=0.525 r_{\mathrm{g}}, 0.538 r_{\mathrm{g}}$, and $0.593 r_{\mathrm{g}}$, respectively

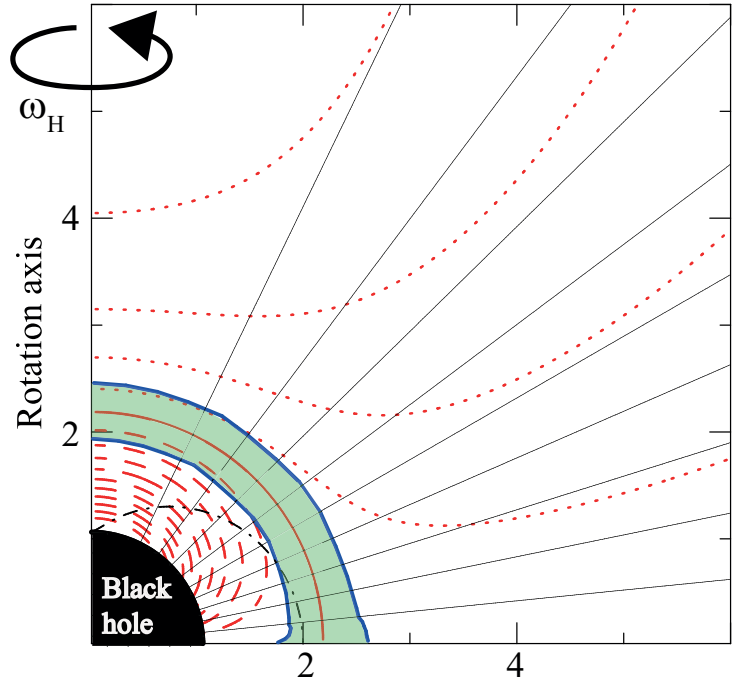

Equatorial plane

FIG. 9.- Similar figure as the left panel of fig. 1 but the gap spatial distribution is depicted as the green-shaded region when the dimensionless accretion rate is $\dot{m}=3.16 \times 10^{-5}$. The gap solution does not strongly depend on the magnetic field configuration such as split-monopole or parabolic ones, because the null surface position is solely determined by frame dragging frequency $\omega(r, \theta)$ and field-line angular frequency $\Omega_{\mathrm{F}}\left(A_{\varphi}\right)$. For example, if $\Omega_{\mathrm{F}}$ is constant for all the magnetic field lines, the null surface distribute nearly spherically as depicted. As $\dot{m}$ decreases further, the inner boundary (thick blue curve that is closer to the black hole) shifts further inwards, and the outer boundary (thick blue curve located away from the hole) shifts further outwards. The split-monopole approximation of the magnetic field lines may be broken down in the lower latitudes, because there is an equatorial accretion flow.

(see also the top panel of fig. 8). Since $\Omega_{\mathrm{F}}=\Omega_{\mathrm{F}}(\Psi)$ is assumed to be constant here, the null surface distributes nearly spherically. In this case, the gap solution little changes in the higher latitudes, because $\rho_{\text {GJ }}$ distribution does not have a strong dependence on $\theta$ in $\theta<30^{\circ}$. Therefore, we assume that the split-monopole magnetic field extends up to $60 r_{\mathrm{g}}$ to compute the pair cascade outside the gap. This treatment is probably justified in the polar funnel of accreting BH systems (McKinney et al. $2007 \mathrm{a}, \mathrm{b})$.

\subsection{Electric field distribution}

Computing the real charge distribution with equation (10), we obtain $E_{\|}$for the critical accretion rate, $\dot{m}=4.87 \times 10^{-6}$, below which no gap solution exists. The result is depicted in figure 10 where the event horizon is located at $s=-1.09 r_{\mathrm{g}}$ on the abscissa. It follows that $E_{\|}$maximizes around the middle point between the horizon and the null surface $(s=0)$. Therefore, a significant fraction of the VHE photons are emitted within $2 r_{\mathrm{g}}<r_{0} \approx 2.1 r_{\mathrm{g}}$ via the curvature process. The maximum attainable Lorentz factor in the middle panel of figure 8 is computed from the solved $E_{\|}(s)$ at each $\dot{m}$, as depicted in the lower panel of figure 10.

\subsection{Emission spectra}

In figure 11, we depict the spectra of the photons emitted by the out-going electrons as thick lines, for three discrete accretion rate, $\dot{m}$. The out-going particles consist of the primary electrons accelerated in the gap and the cascaded pairs outside the gap. These particles up- 


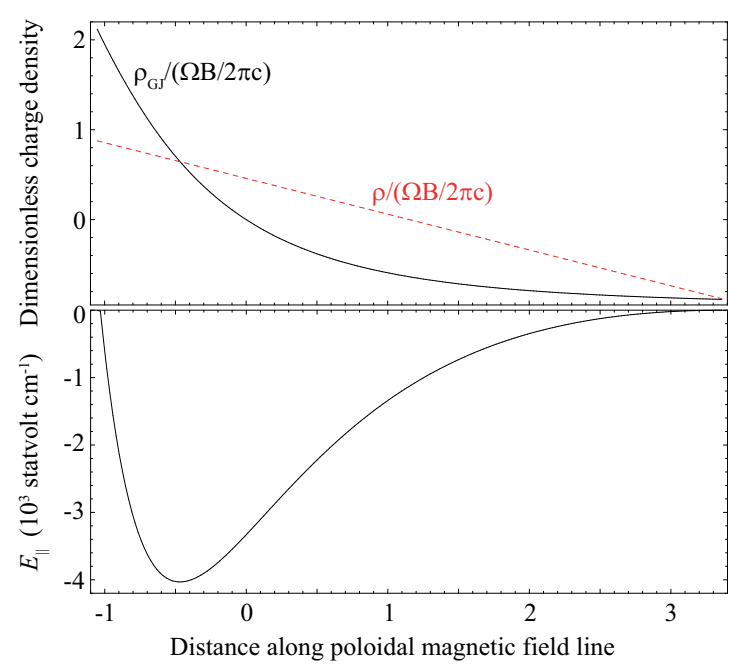

FIG. 10.- Charge densities and acceleration electric field in a non-vacuum gap with maximum screening, $j=1.0$, for the $\mathrm{BH}$ magnetosphere of IC 310. The accretion rate is at the critical value, $\dot{m}=4.87 \times 10^{-5}$. In the abscissa, the inner and outer boundaries are located at $s_{1}=-1.07 r_{\mathrm{g}}$ and $s_{2}=3.38 r_{\mathrm{g}}$, respectively. Top panel: Similar figure as the top panel of figure 4 Bottom panel: Similar figure as the bottom panel of figure 4 but the ordinate is in $10^{3}$ statvolt $\mathrm{cm}^{-1}$ unit.

scatter the background soft photons, which will be partly absorbed by the same soft photon field. The soft photons are provided by the ADAF, whose corresponding spectra (Mahadevan 1997) are indicated by the thin curves. In the $\dot{m} \rightarrow 0$ limit, $d F_{\mathrm{s}} / d E_{\mathrm{s}} \rightarrow 0$ means $w=s_{2}-s_{1} \rightarrow \infty$ in equation (40) in order that $\mathcal{M}=1$ may be held in equation (41). Thus, $r_{0}+s_{1}$ should approach the lower limit, $r_{\mathrm{H}}$, if $\dot{m}$ reduces enough. In the present case, $r_{0}+s_{1}$ reaches $r_{\mathrm{H}}$, when $\dot{m}=4.87 \times 10^{-6}$ (upper panel of fig. 8 ). This most luminous case is depicted as the red thick line in figure 11. We find that the flux of the flare (red filled circles in the inset) can be qualitatively reproduced. It looks contradictory that the gap becomes more luminous with decreasing accretion rate. However, it is a natural consequence of rotation-powered particle accelerator such as in pulsars. In the present case, it is the BH's rotational energy that energizes the gap. It forms a striking contrast to accretion-powered systems.

Figure 11 also shows that the VHE spectrum extends into higher energies with increasing VHE flux. This is because the soft photon density decreases with decreasing $\dot{m}$, thereby reducing the absorption of the primary IC photons above $10 \mathrm{TeV}$. Owing to this photon-photon collisions, there appears a sharp cutoff around 50-100 TeV. In the most luminous case (red solid line), the cutoff appears around $100 \mathrm{TeV}$.

Fermi/LAT observed IC 310 between August 5, 2008 and July 31, 2011, and detected eight photons between $12 \mathrm{GeV}$ and $148 \mathrm{GeV}$ (Aleksic et al. 2014a); the fluxes are plotted by the open circles. Considering the small duty cycle of the IC 310's BH gap, the Fermi/LAT flux, which was averaged over the three years, appears to be higher than our prediction. In addition, the X-ray fluxes, which are represented by the solid and dotted bowties, obviously exceed our prediction. The strong X-ray flux may be due to the emission from a corona or the jet.

In figure 12, we plot the total, primary IC, primary curvature spectra as the thick red solid, black dashed, and black dash-dotted lines for the case of critical accre-

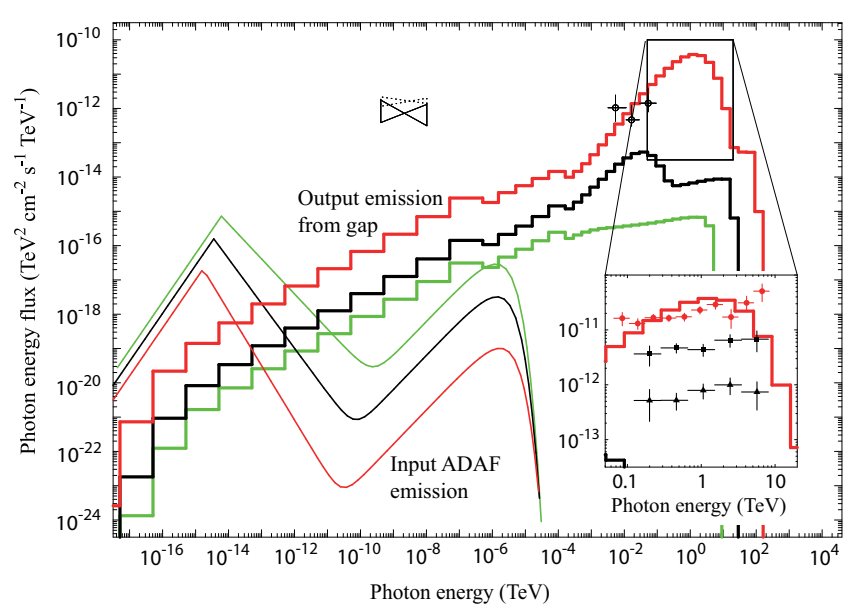

FIG. 11.- Spectral energy distribution of the photons emitted from the gap exerted in the vicinity the supermassive BH of IC 310. The thin green, black and red curves show the input ADAF spectra (Mahadevan 1997) for $\dot{m}=1.0 \times 10^{-4}, 3.16 \times 10^{-5}$, and $4.87 \times 10^{-6}$, respectively. The thick three lines show the output emission of the gap at each accretion rate. The thick red line gives the maximum photon flux, because the gap inner boundary almost touches down the horizon at $\dot{m}=4.87 \times 10^{-6}$. Below this critical accretion rate, gap solution ceases to exist. The magnetic field strength is assumed to be $B=10^{4}\left(r_{0} / r\right)^{2} \mathrm{G}$ at Boyer-Lindquist radius $r$, where $r_{0}$ denotes the radius at the null surface. At greater accretion rate $\dot{m} \sim 1.0 \times 10^{-4}$, the inverse-Compton process dominates the curvature one; thus, the thick green line exhibits a power-law spectrum between $100 \mathrm{MeV}$ and $2 \mathrm{TeV}$. The inset shows a close-up in the VHE regime; the black triangles and squares show the observed fluxes in the low and high states Aleksic et al. 2011) respectively, while the red circles do those in the flaring state (Aleksic et al. 2014b). The observed VHE fluxes are corrected for the absorption by the extragalactic background light. The solid bowtie represents the X-ray fluxes obtained by re-analyzing the observational data taken with XMM-Newton in 2003, Chandra in 2004, and Swift in 2007 (Aleksic et al. 2014a). The dotted bowtie represents the re-analyzed data of Chandra in 2005 (Aleksic et al. 2014a). The open circles denote the Fermi/LAT spectrum.

tion rate, $\dot{m}=4.87 \times 10^{-6}$; the red lines are common in figures 11 and 12. Since the mean-free path of IC scatterings is much longer than $w$, the highest energy photons $(>100 \mathrm{TeV})$ are mostly emitted outside the gap. On the contrary, since the electrons cool down at much shorter interval than $w$ via the curvature process, the curvature photons are mostly emitted inside the gap. These curvature photons dominates the $0.1-10 \mathrm{TeV}$ flux, becoming more and more important with decreasing $\dot{m}$, and hence with increasing output flux. This is because the curvature emission power is proportional to $\gamma^{4}$, while the IC one to $\gamma^{1} \sim \gamma^{2}$ (depending on whether the scatterings take place in the extreme Klein-Nishina or the Thomson limit). Since the absorption works mainly above $10 \mathrm{TeV}$, the curvature photons are little absorbed by the ADAF soft photon field.

The primary IC photons are, on the contrary, heavily absorbed to be reprocessed as the secondary synchrotron and IC components, which are depicted as the dash-dotdot-dotted line. These secondary photons are absorbed again above $10 \mathrm{TeV}$ to be reprocessed as the tertiary component (black dotted line). We compute such a paircreation cascade until the 30th generation, depicting the quaternary and quinary components as the green dotted lines (as labeled with 4 and 5).

Let us briefly examine the case in which $B$ is evaluated by its equilibrium value, equation (24). In figure 13. we 


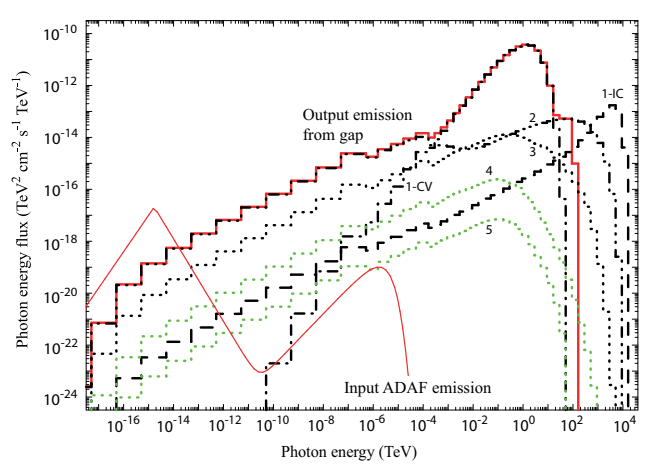

FIG. 12.- Similar figure as figure 11 but only for the case of the critical accretion rate, $\dot{m}=4.87 \times 10^{-6}$, is depicted. The thin and thick red solid lines are identical with those in fig. 2 . The thick dashed line (labeled by 1-IC) represents the flux of the inverse-Compton (IC) photons emitted by the primary electrons, while the dash-dotted line (labeled by 1-CV) represents that of the curvature photons emitted by the primary electrons. The dashdot-dot-dotted line (labeled by 2) shows the sum of the IC and the synchrotron photons emitted by the secondary pairs created outside the gap. The black dotted line (labeled by 3) represents the tertiary photon spectrum, while the green dotted ones (labeled by 4 and 5 ) the quaternary and quinary components.

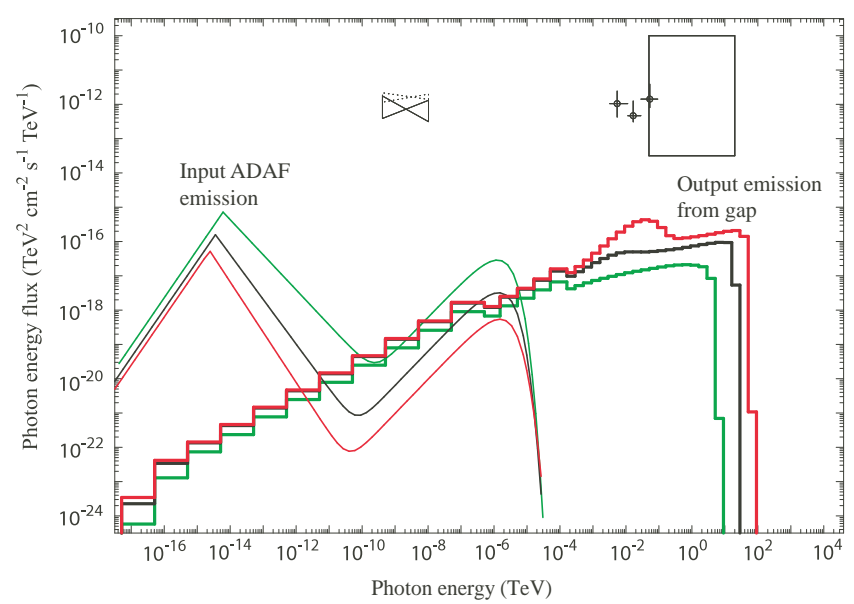

FIG. 13.- Similar figure as fig. 11 but the equilibrium magnetic field strength is used $\left(\S[5.1)\right.$, instead of $B=10^{4}\left(r_{0} / r\right)^{2} \mathrm{G}$. The green and black thin curves represent the input ADAF spectra for $\dot{m}=1.00 \times 10^{-4}$ and $\dot{m}=3.16 \times 10^{-5}$, respectively, whereas the red thin curve does that for $\dot{m}=5.62 \times 10^{-6}$.

present the photons spectra. The input parameters are the same as figure 11. However, the critical accretion rate becomes $\dot{m}=5.62 \times 10^{-6}$ in this case. As analytically expected in $\S 5.1$ the weaker magnetic field, $B=43.3 \mathrm{G}$ at this critical accretion rate, cannot reproduce the observed VHE fluxes at all.

It may be desirable to sum up the main points that have been made in this subsection 5.6.

(1a) The primary electrons (or positrons) are accelerated outward (or inward) by the magnetic-field-aligned electric field.

(1b) The primary particles emit primary photons efficiently via the IC and the curvature processes.

(1c) If these primary photons materialize as pairs in the gap, the created pairs are separated and accelerated to become the primary particles as listed in item (1a).

(1d) The observed VHE flare photons are mostly emitted via the curvature process.

(1e) Although the curvature process dominates the IC one, it is the IC photons that materialize within the gap, colliding with the ADAF submillimeter photons. Thus, both curvature and IC processes are essential, forming a striking contrast to pulsar gap models, in which only the curvature process governs the electrodynamics (because of the higher soft photon energies).

(2a) If these primary photons materialize outside the gap, the created pairs migrate away from the gap as the secondary pairs.

(2b) These secondary pairs emit secondary photons via the IC and the synchrotron processes.

(3a) If these secondary photons materialize within the magnetosphere, the created pairs migrate away from the gap as the tertiary pairs.

(3b) These tertiary pairs emit tertiary photons via IC and synchrotron processes, and so on.

(4) We recur such calculations until the pairs or the photons propagate to $60 r_{\mathrm{g}}$, or until the pairs cascade into the 30 th generation.

\subsection{Created pairs outside gap}

Let us look in more details at the pairs cascaded outside the gap. To examine the most efficient case of pair cascade, we consider the solution at the critical accretion rate, $\dot{m}=4.87 \times 10^{-6}$ for $B=10^{4}\left(r_{0} / r\right)^{2} \mathrm{G}$ (i.e., the red curves in figs. $11 \&$ 12). Figure 14 shows the pairs' energy spectra, integrated over the entire volume of the $\mathrm{BH}$ magnetosphere within $r_{0}+s_{2}<r<60 r_{\mathrm{g}}$, assuming a spherical symmetry. The primary IC photons collide with the radio-submillimeter ADAF photons to materialize as the secondary pairs whose energy spectrum is represented by the red solid line. Such secondary pairs up-scatter the ADAF soft photons that are capable of materializing into the tertiary pairs (black dashed). Such tertiary pairs emit tertiary $\gamma$-rays, some of which materialize as the quaternary pairs (green dash-dotted). At such a low accretion rate as $\dot{m}=4.87 \times 10^{-6}$, pair creation cascade finishes at the quinary generation (blue dotted). The spectrum sharpens with increasing generation. The reasons are twofolds:

(i) The energy of the cascading pairs decreases with increasing generation.

(ii) The higher generation pairs are created at greater $r$ where the ADAF photon intensity reduces by $r^{-2}$ law. Thus, only relatively higher energy pairs can cascade into higher generations, because lower energy pairs can emit only the lower energy IC photons that cannot materialize at greater $r$.

In this way, the energy range is restricted from both higher and lower sides, showing a peaking profile around $100 \mathrm{TeV}$ above the quaternary generation.

Typically speaking, primary IC photons around $10^{4} \mathrm{TeV}$ cascade into $10^{2} \mathrm{TeV}$ pairs, which means that a single primary electron eventually cascade into $\sim 10^{2}$ pairs in the BH magnetosphere. See also LR11 for similar conclusions.

Integrating over the pair energies, we obtain $3.03 \times$ $10^{41}$ ergs s$^{-1}$ as the kinetic luminosity of the created pairs. Since the primary ICS luminosity is $6.97 \times$ $10^{41}$ ergs s $^{-1}, 43 \%$ of the up-scattered photon energy is converted into cascaded pairs outside the gap. However, it is only $0.12 \%$ of the primary curvature luminosity. 


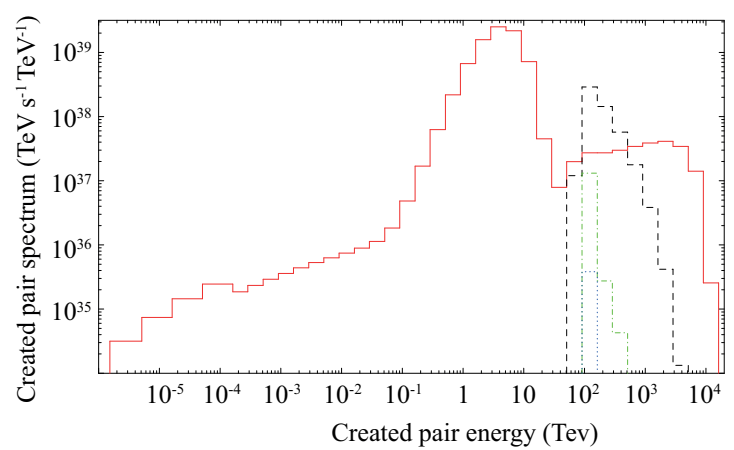

FIG. 14.- Differential pair creation rate within the volume $r<$ $60 r_{\mathrm{g}}$. The red solid, black dashed, green dash-dotted, and blue dotted, lines represent the creation rate of the secondary, tertiary, quaternary, and quinary generation pairs.

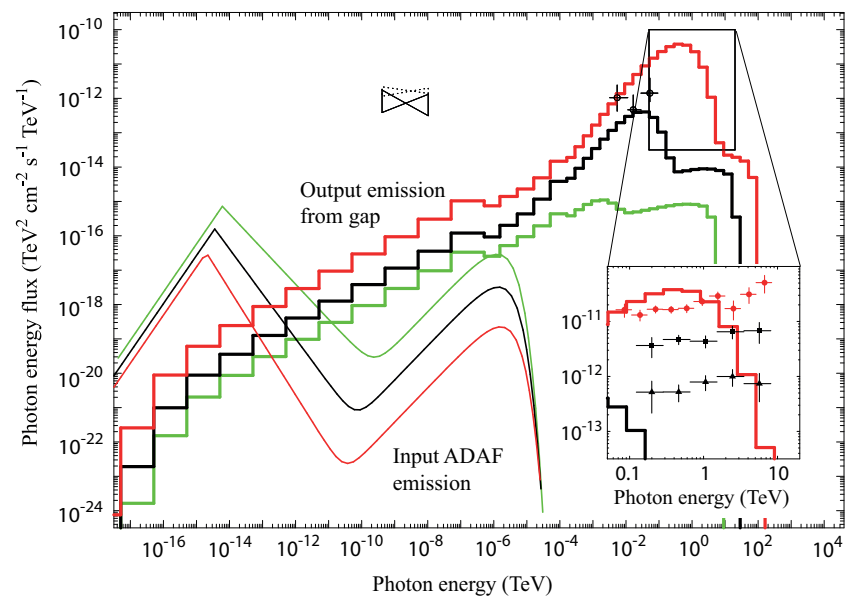

FIG. 15.- Similar figure as figure 11 but a smaller curvature radius, $\rho_{\mathrm{c}}=0.1 r$, is assumed at radius $r$. The green, black and red curves corresponds to the case of $\dot{m}=1.00 \times 10^{-4}, 3.16 \times 10^{-5}$, and $7.50 \times 10^{-6}$ (critical accretion rate), respectively.

\subsection{Dependence on curvature radius}

Let us investigate how the solution depends on the curvature radius, $\rho_{\mathrm{c}}$. In figures 15 and 16, we plot the spectrum obtained for $\rho_{\mathrm{c}}=0.1 r$ and $10 r$ at radius $r$, respectively. The green and black curves represent the cases of $\dot{m}=1.00 \times 10^{-4}, 3.16 \times 10^{-5}$, in the same manner as in figure [11. The red curve corresponds to the critical accretion rate; $\dot{m}=7.50 \times 10^{-6}$ and $\dot{m}=5.62 \times 10^{-6}$ for figures 15 and 16. respectively. It follows that the curvature spectrum, which peaks between 0.1 and $1 \mathrm{TeV}$, becomes softer with decreasing $\rho_{\mathrm{c}}$. This is because equations (30) and (35) give $\nu_{\mathrm{c}} \propto \rho_{\mathrm{c}}^{1 / 2}$, which shows that the curvature-emitted photon energy decreases an order of magnitude if $\rho_{\mathrm{c}}$ decreases from $10 r$ to $0.1 r$. The inset of figure [15] shows that the flare spectrum is difficult to be reproduced above $1 \mathrm{TeV}$, if the curvature radius becomes as small as $\sim 0.1 r$. In this case, electrons' Lorentz factors saturate at one order of magnitude smaller values than the dashed curve in the middle panel of figure 8, because $E_{\|}$in equation (30) depends on $\rho_{\mathrm{c}}$ only weakly in the vicinity of a rotating $\mathrm{BH}$, unlike the situation around a rotating neutron star. Note that $\partial \rho_{\mathrm{GJ}} / \partial s$ is due to the frame-dragging effect in $\mathrm{BH}$ magnetospheres, whereas it is due to the global field line curvature in neutron-star magnetospheres. Thus, $E_{\|}$little depends on $\rho_{\mathrm{c}}$ in $\mathrm{BH}$ magnetospheres.

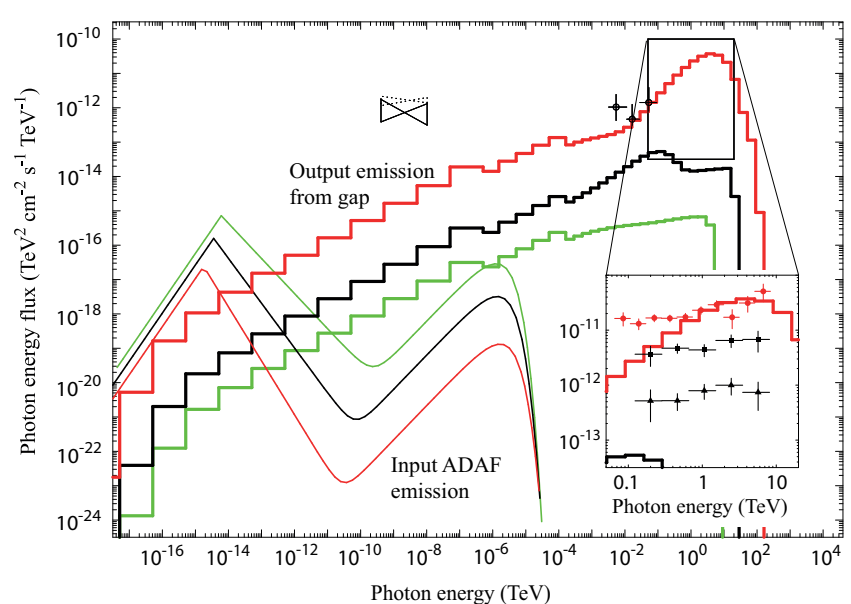

FIG. 16. - Similar figure as figure 11 but a greater curvature radius, $\rho_{\mathrm{c}}=10 r$, is assumed. The green, black and red curves corresponds to the case of $\dot{m}=1.00 \times 10^{-4}, 3.16 \times 10^{-5}$, and $5.62 \times 10^{-6}$, respectively.

In a time-averaged sense, in the polar funnel, the magnetic field lines threading the horizon are wellordered and approximated by a split-monopole configuration (McKinnev et al. 2007a, 2012). Although the split-monopole field is tightly wound azimuthally due to frame dragging near the horizon, it is predominantly radial with loosely wound helics moderately away from the horizon (e.g., at $r>2 r_{\mathrm{g}}$ ) (Hirose et al. 2004). Thus, we consider $\rho_{\mathrm{c}} \sim 10 r$ may be possible in a time-averaged sense.

Despite the constancy of time-averaged magnetized accretion, the instantaneous accretion rate varies considerably with time. In addition, the instantaneous magnetic field lines are kinked and bent in the evacuated funnel, which becomes a cauldron of strong waves (Krolik et al. 2005). Thus, it may be reasonable to consider a superposition of the spectra obtained under different $\rho_{\mathrm{c}}$ 's. For instance, due to a fluctuating magnetic field structure, photons may be emitted into various directions from each position in a time-dependent manner. In such a case, we will detect the combined spectra from different field lines with various $\rho_{\mathrm{c}}$ 's.

As an example, in figure 17. we present the result for the superposition of the $\rho_{\mathrm{c}}=0.1 r$ (blue dotted) and $\rho_{\mathrm{c}}=10 \mathrm{r}$ (red dashed) cases with weight 0.25 and 0.75 , respectively. Since the ADAF photon field may be inhomogeneous (Li et al. 2008), we adopt here different $\dot{m}$ for the $\rho_{\mathrm{c}}=0.1 r$ and $10 r$ cases; namely, $\dot{m}=7.50 \times 10^{-6}$ for the blue dotted line, and $\dot{m}=5.62 \times 10^{-6}$ for the red dashed line. That is, the blue dotted line coincides with the red solid line in figure 15. whereas the red dashed line does the red solid line in figure 16. It follows that a power-law-like spectrum can be formed if curvature spectra with varying $\rho_{\mathrm{c}}$ are superposed with appropriate weights. So that the observed power-law-like VHE spectrum may be reproduced, greater weights are favorable at greater $\rho_{\mathrm{c}}$ 's. In another word, the observed flare spectrum may imply that the instantaneous magnetic field lines are mostly straight but kinked moderately in the funnel. The actual distribution of the weight as a function of $\rho_{\mathrm{c}}$ should be examined separately by numerical analysis. However, it is out of the scope of the present paper. 


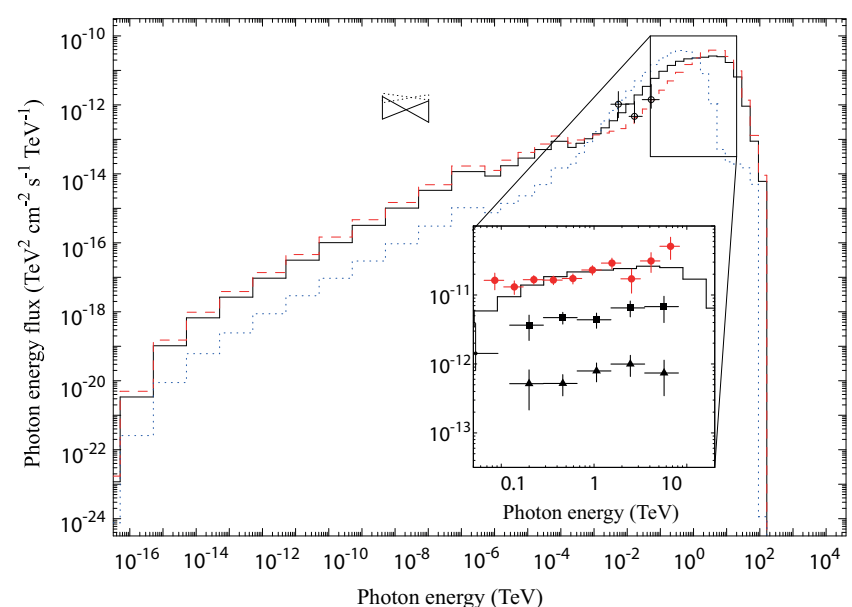

FIG. 17.- Similar figure as figure 11 but the emission spectra with different curvature radius are superposed. The red dashed line shows the spectral energy distribution for $\rho_{\mathrm{c}}=10 \mathrm{r}$, whereas the blue dotted one does one for $\rho_{\mathrm{c}}=0.1 r$. The black solid line shows the superposition of these two curves with weight 0.75 and 0.25 , respectively. In the inset, only the solid line is depicted for clarity. Note that different $\dot{m}$ is adopted for the dashed and dotted lines. See text for details.

\subsection{Dependence on magnetic-field angular frequency}

Let us finally examine the dependence on $\Omega_{\mathrm{F}}$. First, we consider the distribution of the dimensionless GJ charge density, $\rho_{\mathrm{GJ}} /\left(\Omega_{\mathrm{F}} B / 2 \pi c\right)$, on the poloidal plane, assuming a split-monopole magnetic field line configuration. In the left panel of figure [18, we adopt a slower fieldline rotation, $\Omega_{\mathrm{F}}=0.15 \omega_{\mathrm{H}}$. It follows that the null surface (red solid curve) shifts to $r \approx 2.9 r_{\mathrm{g}}$ from $\approx 2.1 r_{\mathrm{g}}$ as $\Omega_{\mathrm{F}}$ reduces to $0.15 \omega_{\mathrm{H}}$ from $0.30 \omega_{\mathrm{H}}$ (cf. left panel of fig. 1). This is because $\omega$ reduces by $r^{-3}$, which indicates that $\omega=\Omega_{\mathrm{F}}$ is realized at greater $r$ for a smaller $\Omega_{\mathrm{F}}$. We plot the (red) dashed contours only below $\rho_{\mathrm{GJ}} /\left(\Omega_{\mathrm{F}} B / 2 \pi c\right)=2.0$, because the gap inner boundary is located in the region where $\rho_{\mathrm{GJ}} /\left(\Omega_{\mathrm{F}} B / 2 \pi c\right)<2.0$ holds, unless a super-GJ electric current is injected across the outer boundary. On the other hand, if the magnetic field lines rotate much faster as $\Omega_{\mathrm{F}}=0.60 \omega_{\mathrm{H}}$, the null surface is located at $r \approx 1.5 r_{\mathrm{g}}$, as the right panel shows. In this case, the inner boundary more easily touches down the horizon, because the GJ charge density at the horizon, $\left|\rho_{\mathrm{GJ}}\right|_{\mathrm{H}}$ is proportional to $\omega_{\mathrm{H}}-\Omega_{\mathrm{F}}$, thereby being limited at a smaller value if $\Omega_{\mathrm{F}}$ approaches $\omega_{\mathrm{H}}$.

Second, we consider the $\gamma$-ray spectrum. At $\Omega_{\mathrm{F}}<$ $0.30 \omega_{\mathrm{H}}$, we can obtain a similar VHE spectrum as the $0.30 \omega_{\mathrm{H}}$ case when the inner boundary does not touches down the horizon, provided that $B$ is unchanged at $10^{4} \mathrm{G}$ in the gap. Alternatively, we obtain a similar VHE spectrum for $B<10^{4} \mathrm{G}$ when the inner boundary touches down the horizon. On the other hand, at $\Omega_{\mathrm{F}}>0.30 \omega_{\mathrm{H}}$, we cannot obtain the observed flare VHE flux even when the inner boundary touches down the horizon, provided that $B=10^{4} \mathrm{G}$. We could adopt $B>10^{4} \mathrm{G}$ and obtain a similar VHE spectrum; however, a greater $B$ would result in a further greater jet-power efficiency (see $\S$ 6.1), which is unfafoured. In figure 19, we present the spectra obtained for $\Omega_{\mathrm{F}}=0.60 \omega_{\mathrm{H}}$ and $B=10^{4} \mathrm{G}$. It shows that the VHE flux reduces significantly and that the curvature spectrum softens if $\Omega_{\mathrm{F}}>0.3 \omega_{\mathrm{H}}$ (cf. fig. 11). Thus, we can conclude that a smaller value of $\Omega_{\mathrm{F}}$ in the polar funnel, which is suggested from numerical and analyti-

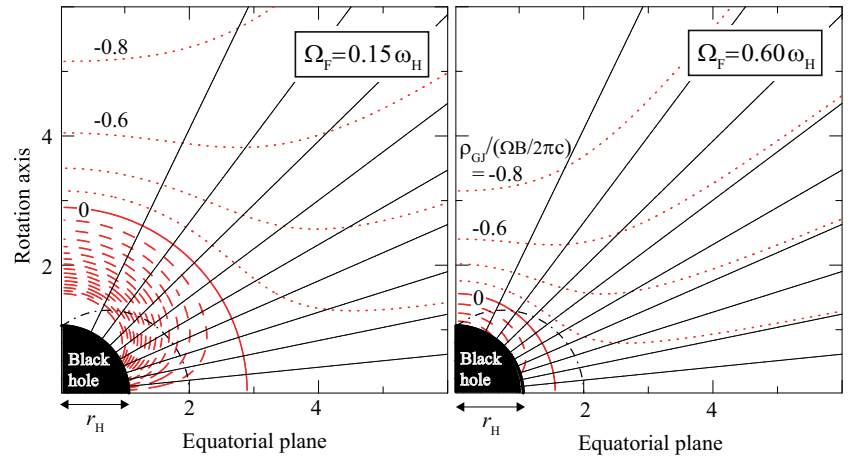

FIG. 18. - Similar figure as the left panel of figure 1] both panels show the dimensionless Goldreich-Julian charge density (red dashed, solid, and dotted contours) for a split-monopole magnetic field (black solid lines). The left panel is for a slower field-line rotation, $\Omega_{\mathrm{F}}=0.15 \omega_{\mathrm{H}}$, while the right panel for a faster rotation, $\Omega_{\mathrm{F}}=0.60 \omega_{\mathrm{H}}$.

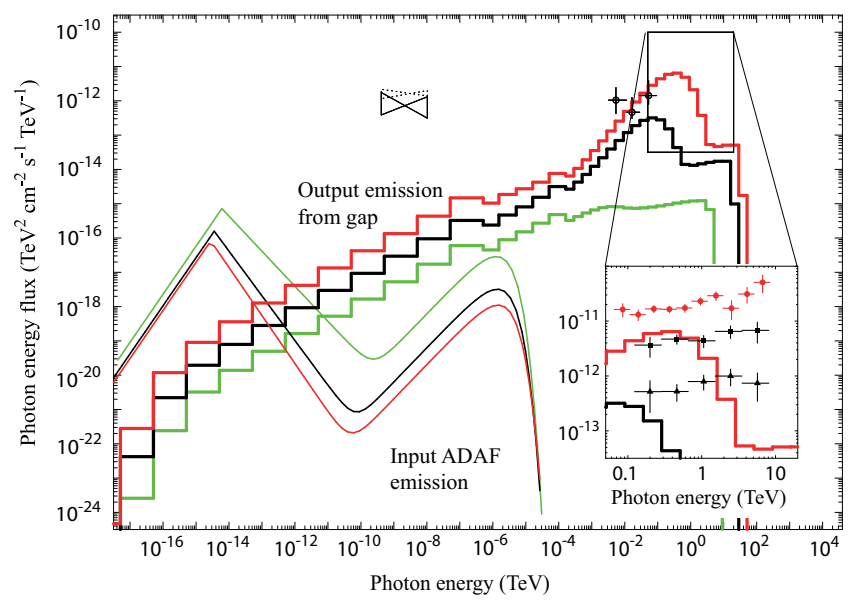

FIG. 19. - Similar figure as figure 11 but a greater rotational angular frequency, $\Omega_{\mathrm{F}}=0.6 \omega_{\mathrm{H}}$, is assumed for the magnetic field. The green, black and red curves corresponds to the case of $\dot{m}=$ $1.00 \times 10^{-4}, 3.16 \times 10^{-4}$, and $1.78 \times 10^{-5}$ (critical accretion rate), respectively.

cal works (McKinney et al. 2012; Beskin \& Zheltoukhov 2013), is favourable to obtain a greater VHE flux from a $\mathrm{BH}$ gap.

\section{DISCUSSION}

In summary, a gap (i.e., a low plasma density region) arises around the null-charge surface that is formed by the frame-dragging effect around a rotating black hole $(\mathrm{BH})$. The gap width along the magnetic field lines, and hence its luminosity increases with decreasing plasma accretion from the surroundings. In the case of IC 310 , the observed jet luminosity and the inferred $\mathrm{BH}$ mass give $\dot{m} \approx 2 \times 10^{-4}$ as the time-averaged, dimensionless mass accretion rate. However, at this accretion rate, the radiatively inefficient accretion flow supplies the electron-positron pairs whose density exceeds the Goldreich-Julian value by an order of magnitude. Thus, in a large fraction of time, the $\mathrm{BH}$ gap is expected to be quenched around the supermassive BH of IC310. Nevertheless, the strong dependence of the created pair density by ADAF photon field (eq. 29) implies the activation of a $\mathrm{BH}$ gap with a small duty cycle by virtue of a timedependent plasma accretion. In the case of IC 310 , it is expected that a $\mathrm{BH}$ gap is activated by a charge deficit if 
the accretion rate is halved from the time-averaged value. If the magnetic field is in equilibrium with the plasma's gravitational biding energy, we obtain $B<10^{3} \mathrm{G}$ for IC 310. However, the predicted gamma-ray flux turns out to be more than two orders of magnitude smaller than the flare flux. Thus, to contrive a stronger magnetic field strength, we assume an extremely rotating $\mathrm{BH}, a=0.998 r_{\mathrm{g}}$. Provided that $B \approx 10^{4} \mathrm{G}$ is maintained intermittently near the horizon, we find that the flare flux can be reproduced when the gap inner boundary nearly touches down the horizon. The TeV spectrum is predicted to extend into higher energies with increasing TeV flux.

\subsection{Limitations of the present model when applied to IC 310}

\subsubsection{Energy extraction efficiency}

When the accretion rate takes its time-averaged value, $\dot{m} \approx 2 \times 10^{-4}$, the radiation inefficient accretion flow (RIAF) supplies copious photons via the free-free process. These MeV photons collide each other so efficiently that the created pair density exceeds the GoldreichJulian (GJ) value. In this case, the hole's rotational energy is extracted via the Blandford-Znajek process (i.e., the electromagnetic energy extraction process in a forcefree magnetosphere) and a steady jet is emitted with luminosity $L_{\text {jet }} \approx 2 \times 10^{42} \mathrm{ergs} \mathrm{s}^{-1}$, which is consistent with the radio observations $(\S[3)$. During this jet phase, we assume that the magnetic field takes the equipartition value (eq. 24) in a time-averaged sense.

On the contrary, when the accretion rate becomes $\dot{m}<10^{-4}$, the magnetosphere becomes charge-starved and the $\mathrm{BH}$ gap is switched on. To explain the enormous flare luminosity, $L \approx 2 \times 10^{44} \mathrm{ergs} \mathrm{s}^{-1}$, we assumed in the present paper that the magnetic field increases about ten times ( to $B \approx 10^{4} \mathrm{G}$ ) intermittently near the null surface. Note that $B$ does not always have to become $\approx 10^{4} \mathrm{G}$ when $\dot{m}$ reduces. Instead, a local and intermittent increase of $B$ to $\approx 10^{4} \mathrm{G}$ (coincidentally when $\dot{m} \ll 10^{-4}$ ) is sufficient for the BH gap to exhibit a flare activity. The extracted power attains $\sim 100$ times greater value than the accretion power (eq. 24), which means an efficiency of $\sim 10 \%$. However, such a high extraction efficiency has not been demonstrated by any numerical simulations so far. For example, McKinney et al. (2012) considered a rapidly rotating $\mathrm{BH}, a \approx 0.9375 r_{\mathrm{g}}$, and showed that the jet efficiency could attain at most $\sim 900 \%$. Thus, by numerical techniques, it is necessary to demonstrate if such an intermittent compilation of a magnetized plasma $\left(B \sim 10^{4} \mathrm{G}\right)$, and the resultant very high efficiency $\left(\sim 10^{4 \%}\right)$, is indeed possible around an extremely rotating $\mathrm{BH}\left(a=0.998 r_{\mathrm{g}}\right)$.

Unless one considers a highly anisotropic emission (e.g., by relativistic beaming), any emission models will encounter the same problem of a huge efficiency $\left(\sim 10^{4 \%}\right)$. It may indicate that the models refuted in Aleksic et al. (2014b), such as the jet-in-jet model (Giannios et al. 2010) or the jet-cloud interaction model (Bednarek \& Protheroe 1997; Barkov et al. 2010, 2012), may be worth reconsidering.

\subsubsection{Photon density constrained by X-ray observations}

In this paper, we have examined the ADAF theory and concluded that the BH magnetosphere of IC 310 becomes charge starved if $\dot{m}<10^{-4}$. To check this logic, in this subsection, we compare the $\mathrm{MeV}$ photon density predicted by ADAF (eq. 26]),

$n_{\mathrm{s}}=5.64 \times 10^{4}\left(\frac{M_{9}}{0.3}\right)^{-1}\left(\frac{\dot{m}}{2 \times 10^{-4}}\right)^{2}\left(\frac{r}{6 r_{\mathrm{g}}}\right)^{-2} \mathrm{~cm}^{-3}$,

with observations.

Unfortunately, there is no direct observations around $\mathrm{MeV}$ for IC 310 along with other low accretion rate $\mathrm{BH}$ systems. Thus, to estimate the $\mathrm{MeV}$ photon density, here we utilize the X-ray observations and extrapolate the spectrum into $\mathrm{MeV}$ energies.

The X-ray spectrum of IC 310 has been obtained between $2 \mathrm{keV}$ and $10 \mathrm{keV}$ with XMM-Newton (MJD 52697, i.e., February 27, 2003), Chandra (MJD 53363, 53456), and Swift-XRT (MJD 54152) (Aleksic et al. 2014a; Sato et al. 2005). The highest X-ray flux $2.5 \times$ $10^{-3} \mathrm{keV} \mathrm{s}^{-1} \mathrm{~cm}^{-2}$ (with photon index $\Gamma=2.01$ ) was obtained by Chandra (MJD 55456), while the lowest one $8.28 \times 10^{-4} \mathrm{keV} \mathrm{s}^{-1} \mathrm{~cm}^{-2}$ (with photon index $\Gamma=2.55$ ) by XMM-Newton. We will consider the both cases in this subsection to estimate the lower and upper bound of the photon number density around $\mathrm{MeV}$.

First, to extrapolate the observed power-law spectrum into $\mathrm{MeV}$, we assume an exponential cutoff above energy $E_{\mathrm{br}}$, in the differential photon number flux,

$$
\frac{d f}{d E}=N E^{-\Gamma} \exp \left(-\frac{E}{E_{\mathrm{br}}}\right) \mathrm{s}^{-1} \mathrm{~cm}^{-2} \mathrm{keV}^{-1},
$$

where the photon energy $E$ is measured in keV. Multiplying $E$ and integrating equation (43) from 2 to $10 \mathrm{keV}$, we obtain the energy flux in $2-10 \mathrm{keV}$,

$$
F_{\mathrm{X}}=N \frac{10^{2-\Gamma}-2^{2-\Gamma}}{2-\Gamma} \mathrm{keV} \mathrm{s}^{-1} \mathrm{~cm}^{-2} \text {. }
$$

Setting $F_{\mathrm{X}}=2.5 \times 10^{-3} \mathrm{~s}^{-1} \mathrm{~cm}^{-2} \mathrm{keV}^{-1}$ and $8.28 \times$ $10^{-4} \mathrm{~s}^{-1} \mathrm{~cm}^{-2} \mathrm{keV}^{-1}$, we obtain

$$
\begin{aligned}
& N=1.55 \times 10^{-3}, \\
& N=1.13 \times 10^{-3},
\end{aligned}
$$

for the Chandra (MJD 55456) and XMM observations, respectively. We estimate the photon number density at $\mathrm{MeV}$ by

$$
n_{\mathrm{s}, \mathrm{obs}} \equiv\left(\frac{d}{r}\right)^{2} \frac{1}{c}\left(E \frac{d f}{d E}\right)_{\mathrm{MeV}},
$$

where $d=81.4 \mathrm{Mpc}$ denotes the distance to IC 310, and

$$
\left(E \frac{d f}{d E}\right)_{\mathrm{MeV}}=10^{-3(\Gamma-1)} N \exp \left(-\frac{E}{E_{\mathrm{br}}}\right) \mathrm{s}^{-1} \mathrm{~cm}^{-2}
$$

denotes the photon number flux at MeV. Thus, the photon number density at $r=6 r_{\mathrm{g}}$ can be estimated to be

$$
n_{\mathrm{s}, \mathrm{obs}}=2.97 \times 10^{16-3 \Gamma} N e^{-E / E_{\mathrm{br}}}\left(r / 6 r_{\mathrm{g}}\right)^{-2} \mathrm{~cm}^{-3} .
$$


For the low X-ray flux case of $X M M$ observation, we obtain

$$
n_{\mathrm{s}, \mathrm{obs}}=8.8 \times 10^{5} e^{-E / E_{\mathrm{br}}}\left(r / 6 r_{\mathrm{g}}\right)^{-2} \mathrm{~cm}^{-3} .
$$

Therefore, we find that the e-folding energy $E_{\mathrm{br}}$ should be less than $370 \mathrm{keV}$ so that $n_{\mathrm{s}, \mathrm{obs}}$ may become less than $n_{\mathrm{s}}$ at the time-averaged accretion rate, $\dot{m}=2 \times 10^{-4}$. On the other hand, for the high X-ray flux case of Chandra (MJD 55456) observation, we obtain

$$
n_{\mathrm{s}, \mathrm{obs}}=4.6 \times 10^{7} e^{-E / E_{\mathrm{br}}}\left(r / 6 r_{\mathrm{g}}\right)^{-2} \mathrm{~cm}^{-3} .
$$

Therefore, we find $E_{\mathrm{br}}<140 \mathrm{keV}$.

The temperature of Comptonizing electrons is estimated to be $T_{\mathrm{e}}=2 E_{\mathrm{br}}$ for an optically thin plasma and $T_{\mathrm{e}}=3 E_{\mathrm{br}}$ for an optically thick one (Petrucci et al. 2010). Thus, we obtain the conservative upper bound, $T_{\mathrm{e}}<1.2 \mathrm{MeV}$ and $T_{\mathrm{e}}<420 \mathrm{keV}$ for the low and high X-ray flux cases, respectively. Considering that the Compton optical thickness is probably thin at small $\dot{m}$, $T_{\mathrm{e}}<740 \mathrm{keV}$ and $T_{\mathrm{e}}<280 \mathrm{keV}$ may be more appropriate as the upper bound.

Adopting an ADAF theory (Mahadevan 1997), one finds $T_{\mathrm{e}}<600 \mathrm{keV}$ at $\dot{m}>10^{-4}$. Therefore, the density of the $\mathrm{MeV}$ photon emitted by ADAF during the jet phase (not during the VHE flare phase) appear to be consistent with the typical $\mathrm{MeV}$ photon density deduced from the X-ray observations during the low X-ray flux phase. However, if we adopt the high X-ray flux phase, the required electron temperature, $T_{\mathrm{e}}<280 \mathrm{keV}$, appears to be too low. In addition, the e-folding energy is empirically deduced to be above $150 \mathrm{keV}$ (i.e., $T_{\mathrm{e}}>300 \mathrm{keV}$ is obtained) commonly in low accretion rate systems (Del Santo 2012 for galactic black holes; Nowak et al. 2011 for Cyg X-1; Malizia et al. 2014 for Seyfert $1 \mathrm{~s})$. Since $T_{\mathrm{e}}$, and hence $E_{\mathrm{br}}$ increases with decreasing $\dot{m}$, the $\mathrm{MeV}$ photon density may increase with decreasing $\dot{m}$ when $\dot{m} \ll 10^{-4}$, although the normalization of the photon number density decreases in X-rays (e.g., in $2-10 \mathrm{keV}$ ). On these grounds, our ansatz of dropping the accretion rate by half in order to create a $\mathrm{BH}$ gap, will be invalidated, if X-ray observations indicate a $\mathrm{MeV}$ photon density that exceeds equation (42) for IC 310 .

What is more, we used $r=6 r_{\mathrm{g}}$ to evaluate $n_{\mathrm{s}}$ and $n_{\mathrm{s}, \mathrm{obs}}$ in this subsection, because the lower-limit radius of the ADAF emission is set to be $r_{\min }=6 r_{\mathrm{g}}$ in the self-similar analytical solution we are adopting (Mahadevan 1997; see also e.g., Narayan et al. 1995; Lasota et al. 1996 for an accordant choice of $\left.r_{\text {min }}\right)$. Note that the radius $r=6 r_{\mathrm{g}}$ gives a conservative estimate (i.e., in this case, higher value) of $n_{\mathrm{s}, \mathrm{obs}}(r)$ than $r=10 r_{\mathrm{g}}$ does (see the third paragraph of $\S$ 4.1). It is, however, possible to compute the RIAF (including ADAF) down to the horizon, if we solve the hydrodynamical equations (Manmoto 2000; Li et al. 2008) or the magnetohydrodynamical equations (Punsly et al. 2009; McKinney et al. 2012) in the Kerr spacetime. For instance, $n_{\mathrm{s} \text {,obs }}$ may increase inwards with $\propto r^{-2}$ also in $r<6 r_{\mathrm{g}}$; in this (simplified) case, we would underestimate $n_{\mathrm{s} \text {,obs }}$ at the gap center $\left(r=2 r_{\mathrm{g}}\right)$ by nine times. An underestimate of $n_{\mathrm{s}, \mathrm{obs}}$, in turn, incurs an optimistic evaluation of the chargestarvation condition, $n_{\mathrm{s} \text {,obs }}<n_{\mathrm{GJ}}$, although the $r$ dependence of $B$ (and hence that of $n_{\mathrm{GJ}}$ ) is non-trivial. If an observation shows that the $\mathrm{MeV}$ photon density gives $n_{\mathrm{s}, \mathrm{obs}}>n_{\mathrm{GJ}}$, gaps cannot be formed around the BH during the period. To constrain the pair-creating photon density more conclusively, simultaneous observations in $\mathrm{TeV}$ and $\mathrm{MeV}$ energies are crucial.

\subsection{Null surface versus stagnation surface}

As noted in $\S 2$, the gap center shift outwards if electrons are injected across the inner boundary. In particular, figure 7 shows that the gap center will shift to $r>6 r_{\mathrm{g}}$ if the injected current attains $\approx \Omega B /(2 \pi)$. Since a low plasma density is expected around the stagnation surface, it is reasonable to consider a gap formation around it under the existence of such a substantial current injection across the inner boundary.

From the analogy with the pulsar outer gap model (Hirotani \& Shibata 2001), we can expect that the BH gap electrodynamics is little affected by the change of the gap position. This is because the gap solution is essentially described by the factor $w / l_{\mathrm{GJ}}$ (e.g., eq. 21), where $l_{\mathrm{GJ}}$ denotes the length scale in which $\rho_{\mathrm{GJ}}$ changes substantially. If the gap is located around the null surface, we can put $l_{\mathrm{GJ}} \approx r_{\mathrm{H}}$; this dimension analysis would give the similar results as we have derived solving the spatial dependence of $\rho_{\mathrm{GJ}}$ explicitly. On the contrary, if the gap is located around the stagnation surface at radius $r$, we can put $l_{\mathrm{GJ}} \approx r$. Thus, if the gap extends enough and $w \sim r$ holds, the potential drop approaches the EMF exerted across the horizon and $L_{\text {gap }}$ approaches $L_{\text {sd }}$, again in the same manner as pulsars. Thus, the results will not change dramatically if the gap center shifts from the null surface to another place, such as the stagnation surface.

In $\S$ 2, we point out the two representative positions for a gap to be formed: the null surface and the stagnation surface. It is, however, worth noting that more than one gap will not be formed along the same magnetic field lines. This is because the pairs cascaded outside one gap will migrate into the other gap to screen the $E_{\|}$there when they polarize. That is, an injection of pairs quenches the gap when one of the charges (e.g., electrons) return, whereas an injection of a completelycharge-separated flow, which consists of only one sign of charges (e.g., positrons), merely shifts the gap position. In the present solutions, the gap around the null surface is not quenching another gap that would arise at another place. The Poisson equation (5) merely does not allow a gap to be formed around other places than the null surface, if there is no current injection across either the inner or the outer boundaries. In subsequent papers, we will examine a gap formation at another positions (e.g., around the separation surface), considering a current injection cross the inner boundary.

\subsection{Comparison with other BH gap models}

In the present paper, we applied our model to IC 310 , while LR11 and BT15 to M87 and SgrA*. Apart from the applied objects, there are important electrodynamical differences among the three works. We discuss this point in this section.

\subsubsection{Comparison with LR11}

Let us compare the present work with LR11. The main difference appears in two points: The dominant emission process and the gap closure condition. 
First, we consider the dominant emission process. To demonstrate the formation of a power-law-like spectrum in VHE, LR11 assumed that the IC process takes place in the Thomson regime and evaluated $\gamma_{\mathrm{IC}}$ by

$$
\gamma_{\text {Thomson }}=\sqrt{\frac{e E_{\|}}{\sigma_{\mathrm{T}} u_{\mathrm{s}}}}
$$

where $\sigma_{\mathrm{T}}$ denotes the Thomson cross section and $u_{\mathrm{s}}$ the soft photon energy density. We should notice here that only the soft photons having energies below $E_{\mathrm{s}}=m_{\mathrm{e}} c^{2} / \gamma$ contribute in the Thomson regime, unless collisions take place with tiny angles. To compute $u_{\mathrm{s}}$, we have to integrate the ADAF photon density up to the energy $E_{\mathrm{s}}$. Therefore, if $E_{\mathrm{s}}$ appears much less than the ADAF peak energy, we obtain $u_{\mathrm{s}} \ll u_{\text {submm }}$, where $u_{\text {submm }}$ denotes the ADAF photon density in submillimeter wavelengths and essentially represent the soft photon density integrated over all the photon energies.

In the present case, $\gamma>3 \times 10^{9}$ (solid curve in the middle panel of figure 8) means that only the ADAF photons with energies $E_{\mathrm{s}}<2 \times 10^{-4} \mathrm{eV}$ contribute in Thomson regime. Since the ADAF spectrum peaks between $10^{-3} \mathrm{eV}$ and $10^{-2} \mathrm{eV}$, we thus obtain $u_{\mathrm{s}} \ll u_{\text {submm }}$. The thin dotted curve in the middle panel of figure 8 is computed with this reduced $u_{\mathrm{s}}$. On the other hand, LR used $u_{\text {submm }}$ to evaluate equation (52), underestimating $\gamma_{\mathrm{IC}}$ such that $\gamma_{\mathrm{IC}}<\gamma_{\text {curv }}$, or equivalently, the the IC process dominates the curvature process.

In short, the leptonic Lorentz factors are turned out to be regulated via the curvature process in the present paper, while LR11 considered that they are regulated via the IC process. To compute the IC-limited Lorentz factors, which is essential to examine the closure condition, we employ the Klein-Nishina cross section taking account of the ADAF spectrum, while LR11 employed the Thomson limit using a single parameter $u_{\text {submm }}$.

Second, we compare the gap closure condition. LR11 considered a necessary condition for a $\mathrm{BH}$ gap to be sustained and imposed that the cascaded pair density should exceed the GJ value outside the gap. They proposed the closure condition, $\mathcal{M}_{\mathrm{LR}}>n_{\text {pair }} /\left(\left|\rho_{\mathrm{GJ}}\right| / e\right)$, where $\mathcal{M}_{\mathrm{LR}}$ denotes the multiplicity of cascaded pairs from a sing primary lepton (their eq. [25]). However, as demonstrated in the bottom panel of figure 8, the cascaded pair density always exceeds the GJ value (horizontal dotted line). Thus, our solutions automatically satisfy the necessary condition proposed by LR11.

Third, we discuss minor differences. For one thing, we consider the gap arising around the null surface, while LR11 around the stagnation surface. Nevertheless, it will not incur a qualitative difference as demonstrated in the pulsar outer gap model (Hirotani \& Shibata 2001). What is more, our results show that the $\gamma$-ray luminosity is proportional to $\left(w / r_{\mathrm{g}}\right)^{4}$ while $\operatorname{LR} 11\left(w / r_{\mathrm{g}}\right)^{2}$; the additional $\left(w / r_{\mathrm{g}}\right)^{2}$ dependence comes from the fact that the electric potential drop in the gap is proportional to $w$ and that the maximum current is also roughly proportional to $w$ (eq. 19). For pulsar out-gap models, the gap is geometrically thin in the transverse direction of the magnetic field; thus, the potential drop is proportional to $\left(w_{\perp} / r_{\mathrm{g}}\right)^{2}$, where $w_{\perp}$ refers to the trans-magnetic-field thickness of the gap. As a result, the gap luminosity is proportional to $\left(w_{\perp} / r_{\mathrm{g}}\right)^{3}$ (Hirotani 2008), because the total current flowing in the gap is proportional to the gap cross section, which is proportional to $w_{\perp}$. However, a BH gap is thin in the longitudinal direction of the magnetic field; thus, the potential drop is proportional to $\left(w / r_{\mathrm{g}}\right)^{3}$, which leads to the $\left(w / r_{\mathrm{g}}\right)^{4}$ dependence of the gap luminosity (eq. 21). One final point is that we explicitly computed the photon energy dependence of the pair creation rate, ICS emissivity, and curvature emissivity, while LR11 adopted a monochromatic approximation for the photon specific intensity.

\subsubsection{Comparison with BT15}

Let us next compare the electrodynamics of this paper with that of BT15. In the latter paper, they neglected the $\rho_{\mathrm{GJ}}$ term in the Poisson equation, their equation (100). We interpret that they assumed a very large created charge density in the gap, that is, $|\rho| \gg\left|\rho_{\mathrm{GJ}}\right|$. The reasons are as follows: If the created charge density were sub-GJ, $\left|E_{\|}\right|$would become less than the vacuum case, leading to $\left|E_{\|}\right|<E_{\perp}\left(w / l_{\mathrm{GJ}}\right)^{2}$, where $E_{\perp}=$ $\Omega_{\mathrm{F}} \varpi B / c$ (i.e., their eq. [1]), $\varpi$ denotes the distance from the rotation axis, $w$ corresponds to $\Delta$ in their notation, and $l_{\mathrm{GJ}}$ the distance in which the GJ charge density changes substantially. In the case of BT15, they assume that a gap is located at the stagnation surface, namely between $r \approx 5 r_{\mathrm{g}}$ and $10 r_{\mathrm{g}}$; thus, $l_{\mathrm{GJ}}>5 r_{\mathrm{g}}$, which becomes $\approx 5 \times 10^{15} \mathrm{~cm}$ for M87. It follows that their solution of $\Delta \approx 4.3 \times 10^{11} \mathrm{~cm}$ (their eq. [57]) gives $E_{\|} \approx \times 10^{-8} E_{\perp}$, about 100 million times smaller than their $E=E_{\perp}$ (their eq. [1]), as long as the created charge is sub-GJ as sketched in figure 7 To overcome difficulty, one must resort on a super-GJ charge density created in the gap, $\rho \gg \rho_{\mathrm{GJ}}$. For example, if the real charge density is $\sim 10^{8}$ times greater than the GJ value, it appears that the Poisson equation might give $E_{\|} \sim E_{\perp}$, that is, their equation (1), although $\Delta / l_{\mathrm{GJ}} \sim 10^{-4}$.

If the created charge density were super GJ, it would distribute as the red solid line in the middle panel of figure 20. However, in this case, $\rho-\rho_{\mathrm{GJ}}$ is positive (or negative) in the inner (or outer) part of the gap, leading to a positive $E_{\|}$, which in tern contradicts with the outwardly decreasing $\rho$. On the contrary, if $\rho$ increases outward, a negative (or positive) $\rho-\rho_{\mathrm{GJ}}$ in the inner (or outer) part of the gap, would lead to a negative $E_{\|}$, which contradicts with the outwardly increasing $\rho$. Thus, we consider that a super-GJ charge creation within the gap is implausible. This is, indeed, the physical reason why all the pulsar gap models treat sub-GJ charge creation within the gap.

On these grounds, we propose to replace their equation (1) with $\left|E_{\|}\right| \approx E_{\perp}\left(w / l_{\mathrm{GJ}}\right)^{2}$, which approximates our equation (12). In addition, we propose to replace their equation (17) with our condition $\mathcal{M}=1$ (eq. 41]), or with $\mathcal{M}_{\mathrm{LR}}>n_{ \pm} /\left(\left|\rho_{\mathrm{GJ}}\right| / e\right)$ of LR11.

\subsubsection{Comparison with HO98}

In HO98, they solved a set of Poisson equation, Boltzmann equations for electrons and positrons, and the radiative transfer equation for a supermassive $\mathrm{BH}$ parameter. They considered an application to quasars and adopted a large accretion rate, $\dot{m} \sim 0.1$. It was demonstrated that a BH gap supply sufficient pairs so that a 


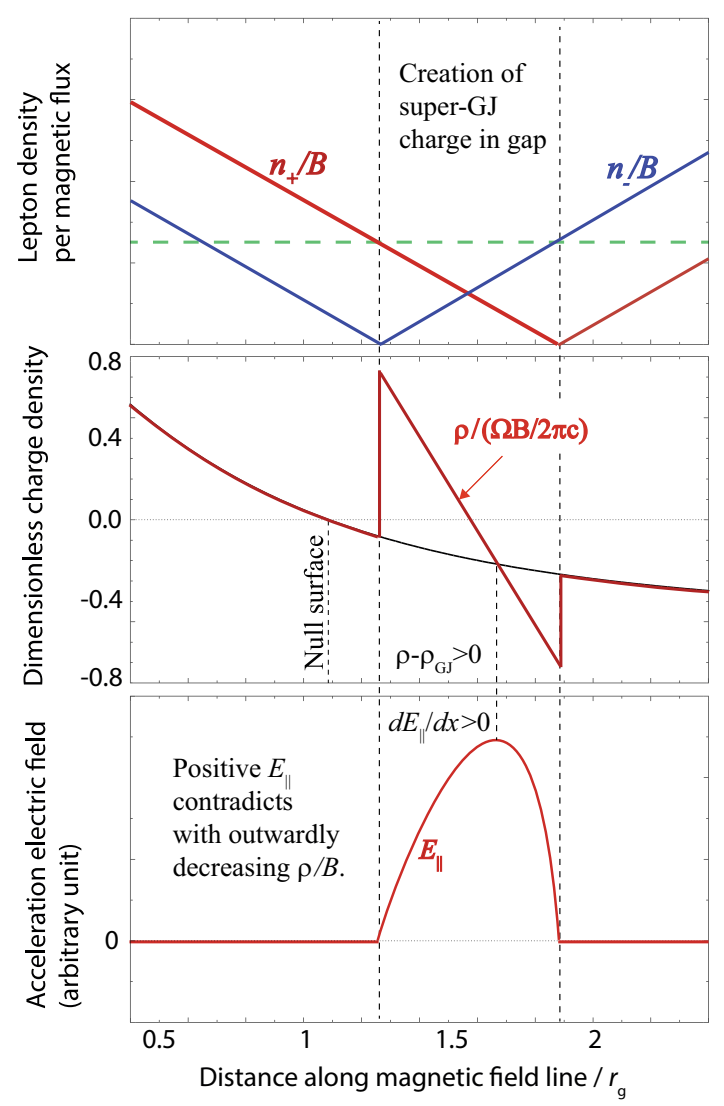

FIG. 20.- Similar figure as figure 4 but the created current density is super-Goldreich-Julian. The gap center is assumed to be remotely located from the null surface.

force-free magnetosphere may be sustained outside the gap, and that the gap luminosity increases with decreasing accretion rate, or the soft photon density. In addition, they concluded that the $\mathrm{BH}$ gap luminosity is totally undetectable, because the gap width is very thin, $w<0.02 r_{\mathrm{H}}$ if $\dot{m} \sim 0.1$. However, they did not notice that a BH-gap emission would be detectable if accretion rate reduces sufficiently.

In the present paper, we therefore consider low luminosity active galactic nuclei and adopted $\dot{m}<10^{-4}$. As the first paper in this series, we only analytically examined the gap closure condition, which is proved to be valid both in $\mathrm{BH}$ magnetospheres (HO98) and in pulsar magnetospheres (Hirotani 2013) through the comparison with the numerical solutions of the set of MaxwellBoltzmann equations.

\subsection{Comparison with pulsar outer-gap model}

Let us compare the present $\mathrm{BH}$ gap model with the pulsar outer-magnetospheric particle accelerator model, that is, the outer-gap (OG) model.

First, in a pulsar magnetosphere, the null surface appears because of the convex geometry of a dipole magnetic field. On the other hand, in a $\mathrm{BH}$ magnetosphere, a null surface appears due to the frame-dragging effect around a rotating black hole. Thus, for the same magnetic field polarity, the sign of $E_{\|}$is opposite. In a $\mathrm{BH}$ magnetosphere, a pulsar-like null surface could also appear far away from the horizon, in principle. However, unless a strong ring current flows within a certain radius, a dipolar field will not be formed in a $\mathrm{BH}$ magne- tosphere. Various numerical simulations (Hirose et al. 2004; McKinnev et al. 2006, 2007a; Tchekhovskov et al. 2010; Punsly et al. 2009; McKinnev et al. 2012) seems to rule out the formation of such a dipolar-like field geometry, except for the initial-condition-dependent closed poloidal field lines in the equatorial torus imposed far away from the horizon.

Second, in the OG model, soft photon field is provided by the cooling neutron star (NS) thermal emission and/or the heated polar-cap thermal emission. The NS surface emissions peak in the X-ray energies. Thus, the curvature 1-10 GeV photons efficiently materialize as pairs within the gap, thereby contributing for the gap closure. For very young pulsars like the Crab pulsar, IC photons could also materialize. However, the thermal IR photon field is much weaker than the thermal X-ray field; thus, the IC photons do not contribute for gap closure in pulsars. In the $\mathrm{BH}$ gap model, it is the ADAF that provides the soft photon field. The ADAF spectrum peaks in submillimeter wavelengths. Thus, the $100 \mathrm{TeV}$ IC photons efficiently materialize as pairs to close the gap. The 0.1-10 TeV curvature photons, on the other hand, do not materialize efficiently, because the IR photon number flux is 5-6 orders of magnitude weaker than the submillimeter one.

Third, if a NS is isolated or is not accreting plasmas from the companion in a binary system, the magnetosphere becomes highly vacuum. The pair density becomes comparable to the GJ value if the space-chargelimited flow is drawn from the NS surface, or becomes much less than the GJ charge value if there is no such a plasma injection. Therefore, a gap is inevitably formed to replenish the charge-starved magnetosphere with a large duty cycle, which is essentially unity. In a black hole magnetosphere, on the other hand, ADAF can supply enough charges not only in the lower latitudes but also in the polar funnel via photon-photon pair production. In the case of $\mathrm{IC} 310$, the $\mathrm{BH}$ gap is, therefore, expected to be intermittent, because the $\mathrm{BH}$ gap can be turned on only when the accretion rate becomes less than half of the time-averaged value.

Fourth, a NS magnetosphere is formed by the electric currents inside the NS, whereas a $\mathrm{BH}$ magnetosphere is formed by those outside the BH. Thus, the magnetic field energy dominates the plasma energy in a NS magnetosphere, whereas it is at most comparable to the plasma's gravitational binding energy in a $\mathrm{BH}$ magnetosphere. Thus, an accreting NS allows accretion along the higherlatitude magnetic field lines that would be open without accretion. This enhanced plasma density quenches an $\mathrm{OG}$, once plasma accretion takes place in a binary system. On the contrary, an accreting $\mathrm{BH}$ allows accretion only in the lower latitudes (i.e., near the equator with a certain vertical height), prohibiting plasma penetration into the funnel region, because the centrifugal-force barrier prevents plasma accretion towards the rotation axis. Thus, even under plasma accretion, a $\mathrm{BH}$ gap can be switched on, provided that the ADAF-supplied pair density (via photon-photon collisions) is small compared to the GJ value.

Fifth and finally, OG distribution is highly nonaxisymmetric for an oblique rotator (i.e., a pulsar). This is because $\rho_{\mathrm{GJ}}$ and hence the null surface distributes highly non-axisymmetrically, reflecting the 
three-dimensional magnetic field geometry. However, $\rho_{\mathrm{GJ}}$ and hence the gap distributes nearly spherically in a $\mathrm{BH}$ magnetosphere, irrespective of the magnetic field geometry (or the magnetic inclination angle), as long as $\Omega_{\mathrm{F}}$ is nearly constant for all the field lines. Therefore, the $\mathrm{BH}$ gap solution, which is governed by the $\rho_{\mathrm{GJ}}$ distribution, little depends on the magnetic field geometry (e.g., split-monopole or parabolic), because $\rho_{\mathrm{GJ}}$ distribution is essentially determined by the frame-dragging effect near the horizon, instead of the functional form of $A_{\varphi}(r, \theta)$, particularly in the higher latitudes (fig. 1).

In addition to the differences point out above, there are, of course, similarities between the present $\mathrm{BH}$ gap model and the OG model, because the basic equations are exactly the same.

The first and foremost, the gap width, and hence the $\gamma$-ray efficiency, which is defined by the ratio between the $\gamma$-ray and spin-down luminosities, increases with decreasing soft photon flux. This is because the pair-production mean-free path increases with decreasing photon flux. For instance, the OG $\gamma$-ray efficiency increases with decreasing NS surface emission, or equivalently with increasing NS age (Hirotani 2013). In the same manner, the $\mathrm{BH}$ gap $\gamma$-ray efficiency increases with decreasing accretion rate of ADAF.

Next, superposition of the curvature radiation from different positions produces a nearly power-law spectrum in a limited energy range. In a pulsar magnetosphere, the caustic effect results a compilation of emission from different altitudes with varying $\rho_{\mathrm{c}}$ and $E_{\|}$. However, in a BH magnetosphere, highly tangled, instantaneous magnetic field lines possibly result in a superposition of the curvature emission from different places with varying $\rho_{\mathrm{c}}$ and $E_{\|}$.

\subsection{General relativistic effects on the accretion flow}

In the present paper, we have employed the Newtonian, self-similar, analytical solution of the ADAF (Mahadevan 1997) to specify the background, soft photon field that illuminates the gap. What is more, for simplicity, we have assumed that the soft photon field is homogeneous within $10 r_{\mathrm{g}}$ and its flux reduces by $r^{-2}$ law outside this radius, and that the collision angles between these soft photons and the hard $\gamma$-rays are isotropic. However, in a realistic $\mathrm{BH}$ magnetosphere, position-dependent specific intensity of the soft photon field is necessary to further quantify the photon-photon absorption as well as the IC scatterings. Thus, the next step may be to incorporate the general-relativistic calculations of radiatively inefficient accretion flows (RIAF). Since the photon distribution is found to be highly inhomogeneous and anisotropic around a rapidly rotating $\mathrm{BH}$ (Li et al. 2009), we may be able to constrain the spin of the individual $\mathrm{BHs}$, if we combine the present method, which specifies the emissivity distribution in the magnetosphere, with a sophisticated, numerical RIAF theory.

One of the authors (K. H.) is indebted to Dr. D. Glawion for valuable discussion on the MAGIC data and to Dr. K. Mannheim for commenting on the draft. This work is supported by the Theoretical Institute for Advanced Research in Astrophysics (TIARA) operated under Academia Sinica, and the Formosa Program between National Science Council in Taiwan and Consejo Superior de Investigaciones Cientificas in Spain administered through grant number NSC100-2923-M-007-001-MY3.

\section{REFERENCES}

Aleksic̀, J. et al. 2010, ApJ723, L207

Aleksic̀, J. et al. 2014, A\&A563, A91

Aleksic̀, J. et al. 2014, Science 346, 1080

Bardeed, J. M. 1970, Nature226, 64

Barkov, M. V., Aharonian, F. A., Bosch-Ramon, V. 2010, ApJ724, 1517

Barkov, M. V., Bosch-Ramon, V. Aharonian, F. A., 2010, ApJ755, 170

Bednarek, W., Protheroe, R. J. 1997, MNRAS287,L9

Beskin, V. S., Istomin, Ya. N., \& Par'ev, V. I. 1992, Sov. Astron. $36(6), 642$

Beskin, V. S., Zheltoukhov, A. A. 2013, Astron. Lett., 39, 215

Blandford, R. D., \& Znajek, R. L. 1976, MNRAS, 179, 433

Boyer, R. H. \& Lindquist, R. W. 1967 J. Math. Phys. 265, 281

Broderick, A. E., Tchekhovskoy A. 2015, ApJ 809, 97

Camenzind, M. A. 1986a, A\&A, 156, 137

Camenzind, M. A. 1986b, A\&A, 162, 32

Cheng, K. S., Ho, C. \& Ruderman, M. 1986, ApJ, 300, 500

Cheng, K. S., Ruderman, M. \& Zhang, L. 2000, ApJ, 537, 964

Del Santo, M. 2012, http://arxiv.org/abs/1209.2880

Eisenacher, D. et al., 2013, Proc. to the 33rd ICRC, Id. 0336, Rio de Janerio, Brazil

Schulz, R., Kadler, M., Ros, E., Eisenacher Glawion D., Bach, U., Elsässer, D., Grossberger, C., Kreykenbohm, I., Mannheim, K., Müller, C., Trüstedt, J., Wilms, J. arXiv:1502.03559

Giannios, D., Uzdensky, D. A., Begelman, M. C. 2010, MNRAS402, 1649

Ghisellini, G. et al. 2014, Nature515, 376

Goldreich, P., Julian, W. H., 1969, ApJ157, 869

Hirose, S. Krolik, J., de Villiers, J.-P., Hawley, J. F. 0224, ApJ606, 1083

Hirotani, K. Takahashi, M., Nitta, S., Tomimatsu, A. 1992, ApJ386, 455
Hirotani, K., Tomimatsu, A., Takahashi, M. 1993, PASJ45, 431

Hirotani, K. \& Okamoto, I. 1998, ApJ, 497, 563

Hirotani, K., \& Shibata, S. 1999, MNRAS308, 67

Hirotani, K., \& Shibata, S. 2001, ApJ558, 216

Hirotani, K., \& Shibata, S. 2001, MNRAS325, 1228

Hirotani, K., Harding, A. K., \& Shibata, S., 2003, ApJ591, 334

Hirotani, K. 2008, ApJ688, L25

Hirotani, K. 2006b, Mod. Phys. Lett. A (Brief Review) 21, 1319

Hirotani, K. 2011, ApJ733, L49

Hirotani, K. 2013, ApJ766, 98

Hirotani, K. 2015, ApJ798, L40

Ichimaru, S. 1979, ApJ214, 840

Jauch, J. M., \& Rohrlich, F. 1955, Theory of photons and electrons (Addison-Wesley Publishing Inc., Massachusetts)

Kerr, R. P. Phys. Rev. Lett.11, 237

Koide, S. et al. 1976, MNRAS, 179, 433

Krolik, J. H., Hawley, J. F., Hirose, S. 2005, ApJ, 622, 1008

Lasota, J. P., Abramowicz, M. A., Chen, X., Krolik, J., Narayan, R., \& Yi, I. 1996, ApJ462, 192

Levinson, A., Rieger, F. 2011, ApJ730, 123

Li, Y.-R., Yuan, Y.-F., Wang, J.-M., Wang, J.-C., Zhang, S. 2008, ApJ699, 513

Mahadevan, R. 1997, ApJ477, 585

Malizia, A., Molina, M., Bassani, L., Stephen, J. B., Bazzano, A., Ubertini, P., Bird, A. J. 2014, ApJ782, L25

Manmoto, T., Mineshige, S., Kusunose, M. 1997, ApJ489, 791

Manmoto, T. 2000, ApJ534, 734

Merloni, A., Heinz, S., di Matteo, T. 2003, MNRAS345, 1057

McElroy, D. B., 1995, ApJS100, 105

McKinney, J. C. 2006, MNRAS368, 1561

McKinney, J. C., Narayan, R. 2007a, MNRAS375, 513

McKinney, J. C., Narayan, R. 2007b, MNRAS375, 531 
McKinney, J. C., Tchekhovskoy, A., Blandford, R. D. 2012, MNRAS423, 3083

Mestel L. 1971, Nature 233, 149

Michel F. C. 1973, ApJ180, L133

Nakamura, K. E., Matsumoto, R. Kusunose, M., Kato, S. 1997, PASJ49, 503

Narayan, R., Yi, I. 1994, ApJ428, L13

Narayan, R., Yi, I. 1995, ApJ452, 710

Narayan, R., Yi, I., Mahadevan, R. 1995, Nature374, 623

Narayan, R., Kato, S., Honma, F. 1997, ApJ476, 49

Neronov, A., Semikov, D., Vovk, I. 2010, A\&A519, L6

Nowak, M. A., Hanke, M., Trowbridge, S. N., Markoff, S. B. Wilms, J., Pottschmidt, K., Coppi, P., Maitra, D., Davis, J. E.; Tramper, F. 2011, ApJ728, 13

Petrucci, P. O., Haardt, F. Maraschi, L. et al. 2010, ApJ721, 1340

Phinney, S. 1983, unpublished PdH thesis, Cambridge University

Punsly, B., Igumenshchev, I. V., Hirose, S. 2009, ApJ704, 1065

Punsly, B. 2011, MNRAS418, L138
Rhee, G., Burns, J. O., Kowalski, M. P. 1994, AJ108, 1137

Romani, R. 1996, ApJ470, 469

Li, R.-Y., Yuan, Y.-F., Wang, J.-M., Wang, J.-C., Zhang, S. 2009, ApJ513, 524

Rybicki, G. B. \& Lightman, A. P. 1979, Radiative Processes in Astrophysics (New York: John Wiley \& Suns)

Sato, K. et al., 2005, PASJ57, 743

Schwarz, R. A. et al. 1992, A\&A256, L11

Sijbring, D., \& de Bruyn, A. G. 1998, A\&A331, 901

Sikora, M., Begelman, M. C. 2013, ApJ764, L24

Simien, F., Prugniel, P. 2002, A\&A384, 371

Spitkovsky, A. 2006, ApJ648, L51

Takahashi, M., Nitta, S., Tatematsu, Y., Tomimatsu, A. 1990, ApJ363, 206

Tchekhovskoy, A., Narayan, R., McKinney, J. C. 2010, ApJ711, 50

Thorne, K. S. 1974, ApJ191, 507 